ARTICLE

https://doi.org/10.1038/s41467-019-10226-9

\title{
DRP1-mediated mitochondrial shape controls calcium homeostasis and muscle mass
}

\author{
Giulia Favaro 1,2,11, Vanina Romanello 1,2,3,11, Tatiana Varanita4, Maria Andrea Desbats ${ }^{5,6}$, Valeria Morbidoni ${ }^{5,6}$, \\ Caterina Tezze 1,2, Mattia Albiero (1) 1,7, Marta Canato², Gaia Gherardi², Diego De Stefani², Cristina Mammucari², \\ Bert Blaauw ${ }^{1,2,3}$, Simona Boncompagni ${ }^{8}$, Feliciano Protasi ${ }^{8}$, Carlo Reggiani ${ }^{2,9}$, Luca Scorrano (i) ${ }^{1,4}$, \\ Leonardo Salviati ${ }^{5,6} \&$ Marco Sandri, $1,3,10$
}

Mitochondrial quality control is essential in highly structured cells such as neurons and muscles. In skeletal muscle the mitochondrial fission proteins are reduced in different physiopathological conditions including ageing sarcopenia, cancer cachexia and chemotherapyinduced muscle wasting. However, whether mitochondrial fission is essential for muscle homeostasis is still unclear. Here we show that muscle-specific loss of the pro-fission dynamin related protein (DRP) 1 induces muscle wasting and weakness. Constitutive Drp1 ablation in muscles reduces growth and causes animal death while inducible deletion results in atrophy and degeneration. Drp1 deficient mitochondria are morphologically bigger and functionally abnormal. The dysfunctional mitochondria signals to the nucleus to induce the ubiquitin-proteasome system and an Unfolded Protein Response while the change of mitochondrial volume results in an increase of mitochondrial $\mathrm{Ca}^{2+}$ uptake and myofiber death. Our findings reveal that morphology of mitochondrial network is critical for several biological processes that control nuclear programs and $\mathrm{Ca}^{2+}$ handling.

\footnotetext{
${ }^{1}$ Venetian Institute of Molecular Medicine, via Orus 2, 35129 Padova, Italy. ${ }^{2}$ Department of Biomedical Science, University of Padova, via G. Colombo 3, 35100 Padova, Italy. ${ }^{3}$ Myology Center, University of Padova, via G. Colombo 3, 35100 Padova, Italy. ${ }^{4}$ Department of Biology, University of Padova, via U. Bassi 58B, 35121 Padova, Italy. ${ }^{5}$ Clinical Genetics Unit, Department of Woman and Child Health, University of Padova, via Giustiniani 3,35128 Padova, Italy. ${ }^{6}$ IRP Città della Speranza Corso Stati Uniti 4, 35127 Padova, Italy. ${ }^{7}$ Department of Medicine - DIMED, University of Padova, 35128 Padova, Italy. ${ }^{8}$ Center for Research on Ageing and Translational Medicine (CeSI-MeT)), via Luigi Polacchi, University G. d' Annunzio, 66100 Chieti, Italy. ${ }^{9}$ Institute for Kinesiology Research, Science and Research Center of Koper, Koper, Slovenia. ${ }^{10}$ Department of Medicine, McGill University, Montreal, Canada. ${ }^{11}$ These authors contributed equally: Giulia Favaro, Vanina Romanello. Correspondence and requests for materials should be addressed to L.S. (email: leonardo.salviati@unipd.it) or to M.S. (email: marco.sandri@unipd.it)
} 
T he maintenance of a functional mitochondrial network is particularly important for tissues that are highly structured and metabolically active, such as neurons, cardiac, and skeletal muscles. These tissues are constituted by post-mitotic cells that do not divide. As opposed to the tissues with high cell turnover that can dilute the damaged mitochondria among the dividing cells, the post-mitotic tissues use the fusion/fission machinery to preserve or restore mitochondrial function. Eventually, mitochondria that are irreversibly damaged are removed by the autophagy system ${ }^{1}$. Mitochondrial morphology and the number depend on the balance between two opposing processes, fusion and fission. Fusion induces the formation of an extended interconnected mitochondrial network enabling the mitochondria to mix and re-distribute their contents of metabolites, proteins, and mitochondrial DNA (mtDNA) ${ }^{2}$. An interconnected mitochondrial network prevents the local accumulation of defective/ abnormal mitochondria which is advantageous under conditions of high energy demand, such as exercise ${ }^{3,4}$. Conversely, mitochondrial fission or fragmentation is a mechanism that segregates components of the mitochondrial network, which are dysfunctional or damaged, allowing their removal via mitophagy $y^{5,6}$. Hence, dynamic regulation of the fission-fusion events adapts mitochondrial morphology to the bioenergetic requirements of the cell. A failure of these systems predisposes to organ dysfunction and degeneration.

Mitochondrial fission depends on the cytosolic GTPase dynamin-related protein 1 (DRP1), which is recruited to the outer mitochondrial membrane and oligomerizes to form active GTPdependent mitochondrial fission sites ${ }^{7}$. The fission machinery is critical for tissue development and function, and indeed constitutive $\operatorname{Drp} 1$ knockout animals are embryonically lethal ${ }^{8}$. Consistent with this crucial role of mitochondrial dynamics in postmitotic organs, conditional deletion of $\operatorname{Drp} 1$ in the brain leads to perinatal lethality due to brain hypoplasia ${ }^{8}$. The severe phenotypes of these mice preclude studies of mitochondrial function in adult organs ${ }^{8,9}$.

Striated muscles are post-mitotic tissues that rapidly respond to metabolic challenges, like exercise, feeding, or fasting. The plasticity of the mitochondria is a key factor in myofiber metabolic adaptation to different conditions ${ }^{6}$. Alterations in the content, shape, or function of the mitochondria are associated with muscle wasting. Overexpression of the mitochondrial fission machinery in adult muscle is sufficient to induce muscle atrophy ${ }^{10,11}$. In elderly individuals, mitochondria are abnormally enlarged, more rounded in shape, and display matrix vacuolization and shorter cristae $^{12}$. We have recently found that fusion and fission proteins are greatly reduced in atrophic muscles of old sedentary people while they are normally expressed in hypertrophic muscles of senior sportsmen ${ }^{13}$. Similarly, DRP1 protein is reduced in cardiac and skeletal muscles during ageing in mice ${ }^{14}$. Consistently, a transcriptomic and proteomic study in rats has revealed a correlation between reduced expression of fusion/fission proteins and age-related muscle loss ${ }^{15}$. The decrease of fusion and fission proteins happens also in several inherited and acquired muscle diseases. In cancer cachexia and in chemotherapy-induced cachexia, DRP1, OPA1, and Mitofusin2 are dramatically suppressed $^{16-18}$. The same pattern of reduction has been found in an animal model of Myasthenia gravis ${ }^{19}$. Finally, loss-of-function mutation in the DRP1 receptor, MiD49, causes severe myopathy in humans ${ }^{20}$. Altogether these observations suggest that changes of mitochondrial shape might be an important player in determining muscle mass maintenance, homeostasis, and metabolism. However, little is known about the requirement of mitochondrial fission and the downstream signaling in skeletal muscle in vivo. To understand the physiological relevance of the fission machinery in muscle homeostasis, we generate two mouse models lacking Drp1 in skeletal muscle and show that these mice develop a lethal mitochondrial myopathy caused by activation of protein breakdown, ER stress, hypoglycemia, and abnormalities in $\mathrm{Ca}^{2+}$ homeostasis.

\section{Results}

Muscle-specific Drp1 deletion results in postnatal lethality. Because DRP1 level decreases in muscle during ageing and in different conditions of muscle wasting ${ }^{13,14,16-18,20}$, we generated a muscle-specific Drp1 knockout mouse by crossing the Drp $1^{f l f f l}$ mice with MLC1f-Cre transgenic mice $\left(\operatorname{Drp} 1^{-1-}\right)$, where Cre recombinase is expressed in differentiated muscle cells and not in muscle stem cells or in myocytes ${ }^{21}$. Muscles of $D r p 1^{-1-}$ newborns showed a decrease of DRP1 transcript and protein level (Fig. 1a, b; Supplementary Fig. 1a-c), while it was normally expressed in other tissues, including the heart (Fig. 1b). Immunofluorescence analyses confirmed the absence of DRP1 protein in myofibers irrespectively of the type of muscle (Supplementary Fig. 1a, b), while interstitial cells, skin, and vessels do express DRP1 and contribute to the protein detected in western blot of Fig. 1b. Importantly, these mice died within 30 days of postnatal life (Fig. 1c). During the first 17 days of postnatal life, the body growth was reduced in Drp1 $1^{-1-}$ mice (Fig. 1d, e). Muscles displayed a reduction in the total number of fibers (Supplementary Fig. 1e) because glycolytic type $2 \mathrm{~B} / 2 \mathrm{X}$ fibers are less than controls (Fig. 1f) and a concomitant decrease of the cross-sectional area (Fig. 1g). The reduced number of fibers prompted us to analyze myogenesis. However, MyoD and myogenin expression, two markers of muscle stem cell proliferation and differentiation, did not differ between $D r p 1^{-1-}$ and control mice (Fig. 1h; Supplementary Fig. 1d), suggesting that myogenesis is not perturbed at neonatal stage despite postnatal myofiber growth is affected by Drp1 deletion.

Drp1 deletion impairs mitochondrial function. To understand whether DRP1 inhibition altered mitochondria network, we monitored the mitochondrial ultrastructure by electron microscopy (EM) (Fig. 2a). The quantitative EM analysis revealed that, while the relative volume occupied by the mitochondria was not significantly different in DRP1-deficient muscle (Supplementary Fig 2a), the average size of individual organelles was larger in Drp1 $1^{-1-}$ muscle fibers than controls. These findings indicate that DRP1 ablation results in remodeling of the mitochondrial network with bigger, but fewer, mitochondria with no changes in the relative fiber volume occupied by the mitochondrial network (Supplementary Fig. 2a). Consistently, the mitochondrial DNA content was not changed between the two genotypes (Fig. 2b), suggesting that the total mitochondrial content was not decreased. Similarly, mitochondrial mass revealed by TOM 20 and porin proteins was not affected by DRP1 deletion (Fig. 2c; Supplementary Fig. 2e). Lack of DRP1 induced an upregulation of the profusion genes Pgcla, Mfnl, and Opal (Fig. 2d) that may contribute to the observed increase of the mitochondrial volume. $\mathrm{SDH}$ staining of neonatal muscles confirmed the presence of smaller fibers containing an abnormal mitochondrial network with bigger and fewer puncta in $\operatorname{Drp} 1^{-/-}$(Fig. 2e). Functionally, respiration was reduced in the mitochondria purified from Drp $1^{-/}$muscles (Fig. 2f; Supplementary Fig. 2b) being complexI-driven respiration the most severely affected. Consistent with the respiration data, the measurements of complexes activity showed a significant reduction of complex-I and III (Supplementary Fig. 2c). Immunoblots of WT and Drp1 $1^{-/-}$mitochondrial proteins revealed a downregulation in the levels of the tested respiratory chain complexes subunits (Supplementary Fig. 2d). Finally, supercomplexes, separated by Blue Native PAGE, were 

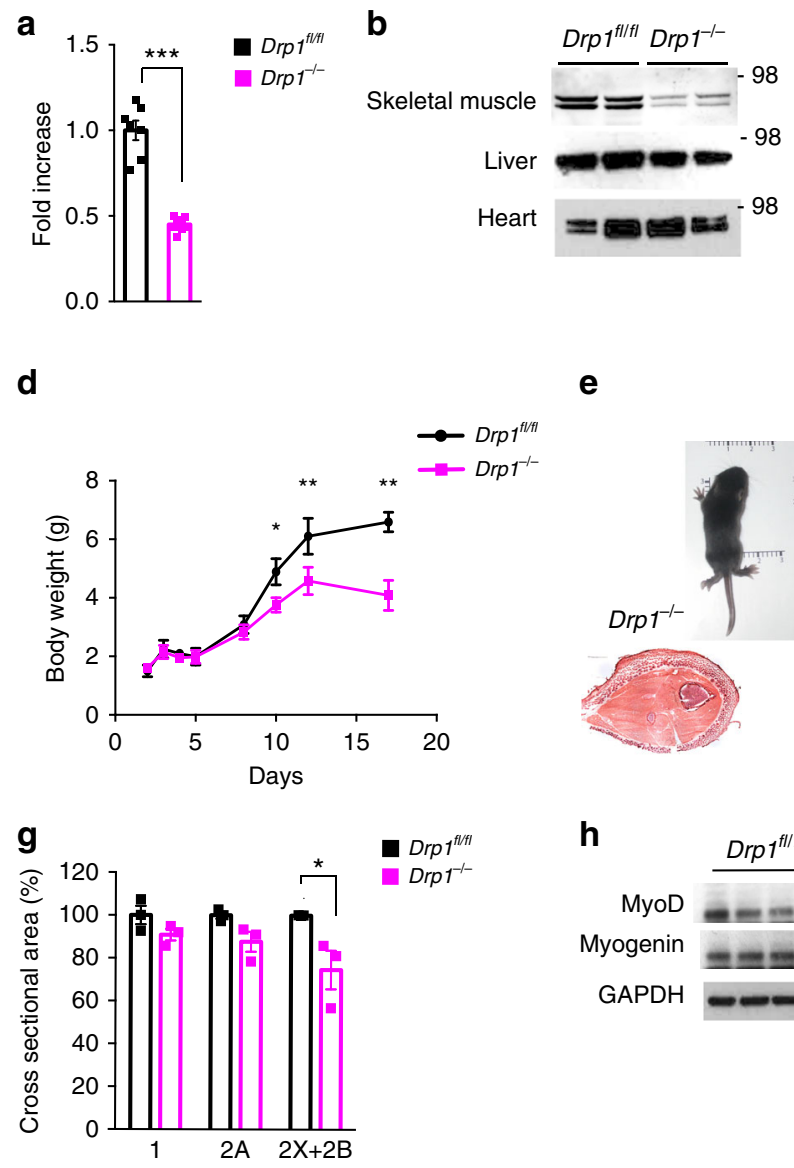

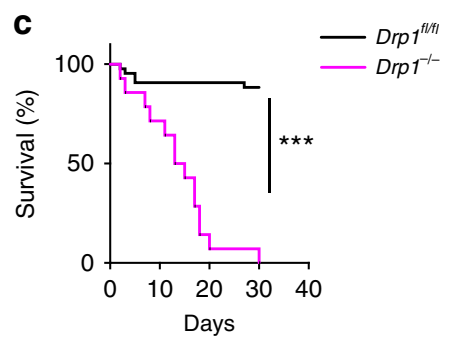

e

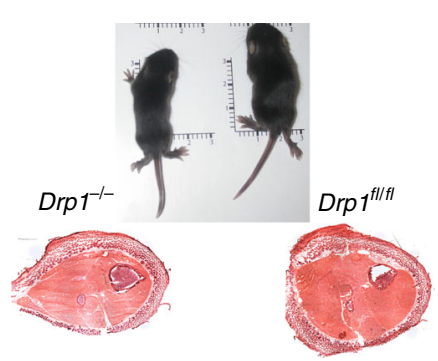

h

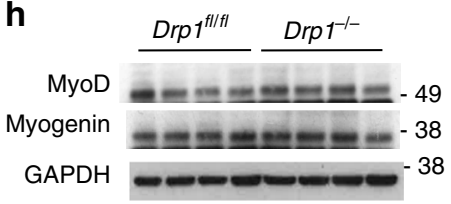

$\mathbf{f}$

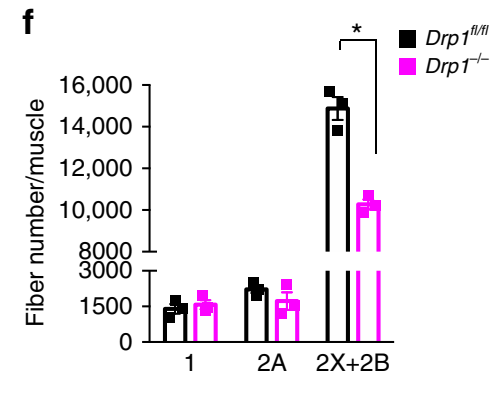

ig. 1 DRP1 deletion impairs muscle growth and causes a lethal phenotype. Drp1 mRNA (a) and DRP1 protein levels (b) are downregulated only in skeletal muscle and not in other tissues in DRP1-null mice. Each condition represents the average of at least three independent experiments \pm SEM. $\mathbf{c}$ Kaplan-Meier survival curve of $\operatorname{Drp}^{f / / f l}$ and $D r p 1^{-/-}$littermates $(W T, n=43 ; K O, n=14$ ), indicates that muscle-specific DRP1 deletion results in lethality within postnatal day 30. d DRP1-deficient mice show an impairment of total body weight. e Upper panel: representative photograph of control and KO littermates at postnatal day 12 showing that Drp1-/- mice are smaller than controls. Lower panel: haematoxilin-eosin (HE) staining of hindlimb cross-section. The crosssectional area analysis of fibers $(\mathbf{g})$ and the total number of different fiber types in hindlimb muscles (f) indicate a reduction of DRP1 KO fiber size without hypoplasia. The data represent mean $\pm \operatorname{SEM}(\mathbf{g}, n=3$ and $\mathbf{f}, n=3$ ). $\mathbf{h}$ Representative immunoblot analysis of muscle homogenates of four independent experiments. MyoD and Myogenin were normalized to GAPDH expression levels. The data represent average \pm SEM. Two-tailed unpaired Student's $t$ test was used. Statistical significance: ${ }^{\star} p \leq 0.05 ;{ }^{\star \star} p \leq 0.01 ;{ }^{\star \star \star} p \leq 0.001$

decreased (Fig. 2g; Supplementary Fig. 2f). Since supercomplex formation is regulated by OPA1 levels ${ }^{22}$, we tested OPA1 and showed that its processing was increased in the $\operatorname{Drp} 1^{-/-}$mitochondria, where short isoforms accumulated (Fig. $2 \mathrm{~h}$ ) in a picture similar to cells where DRP1 is chronically depleted ${ }^{23}$. Therefore, DRP1 ablation alters mitochondrial morphology and function.

Drp1 deletion reduces protein synthesis and induces atrophy. The observed mitochondrial dysfunction does not mechanistically explain the impaired animal growth of $\operatorname{Drp}^{-/-}$mice. During the first 2 weeks of postnatal life, muscle growth depends on fusion of muscle stem cell to the growing myotube and on protein synthesis ${ }^{24}$. Since muscle stem cell differentiation was not perturbed during neonatal growth (Fig. 1h; Supplementary 1d), we monitored protein synthesis in vivo by using the SUnSET technique ${ }^{25}$. Importantly, protein synthesis was reduced by $30 \%$ in $\operatorname{Drp1}^{-1-}$ mice (Fig. 3a; Supplementary Fig. 3a). We therefore turned to pathways that regulate translation. Insulin signaling, through mTOR, plays a critical role in controlling protein synthesis. While AKT phosphorylation was not affected, two mTOR downstream targets were changed, but in opposite pattern. One mTORdependent phosphorylation site of 4EBP1 (Thr 37/46) was increased, while a second one (Ser 65) showed a trend of increase.
Conversely, a second downstream mTOR target, S6, was less phosphorylated in $\operatorname{Drp} 1^{-1-}$ (Fig. 3b; Supplementary Fig. 3b). Thus, the AKT/mTOR/4EBP1/S6 axis is not consistent and does not explain the reduction of protein synthesis, suggesting that other regulators are involved in the translation impairment. The unfolded protein response pathway through the kinase PERK phosphorylates eIF2a, a critical ribosome assembly initiation factor. When eIF2 $\alpha$ is phosphorylated, the ribosomal $43 \mathrm{~S}$ complex formation is decreased, ribosome assembly is impaired, and general protein synthesis is reduced. Interestingly, levels of peIF2 $\alpha$ and of the chaperone Bip/Grp78, a downstream target of UPR, were increased in $\operatorname{Drp}^{-/-}$muscles (Fig. 3c; Supplementary Fig. 3b). Moreover, PERK-dependent UPR triggers the activation of the transcription factor ATF4 that transcribes several genes, including BiP, GADD34, and CHOP. All these genes, including $A T F 4$, were induced in knockout mice, supporting the concept that UPR is strongly activated in $\operatorname{Drp1}^{-/-}$muscles and can account for the translation impairment (Fig. 3d). Consistently, FGF21, a fasting-mimetic cytokine under ATF4 regulation, was induced (Fig. 3e), resulting in increased FGF21 blood levels and decreased glycemia in fed $\operatorname{Drpl}^{-1-}$ mice (Fig. 3f, g). Because FGF21 causes GH resistance, we tested liver expression of IGF1, the critical anabolic hormone that controls the tissues size during 
a

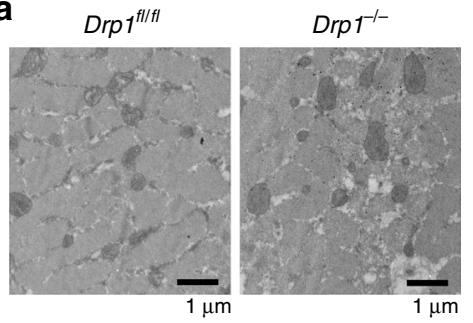

d

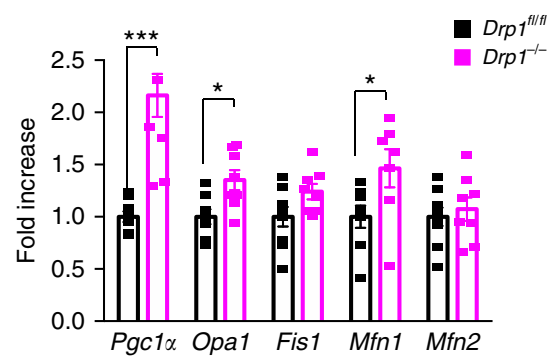

b

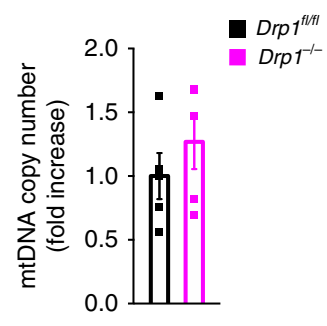

C

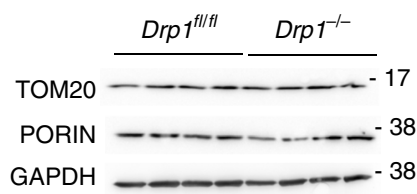

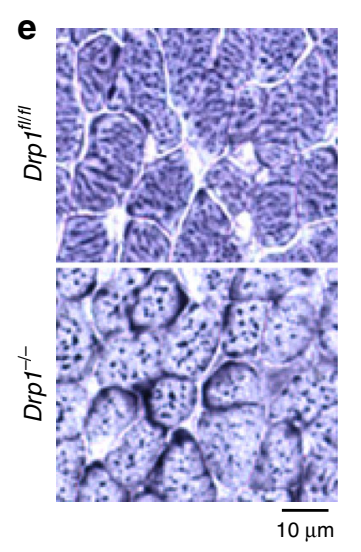

f

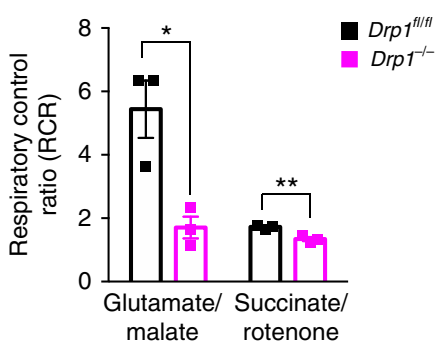

h
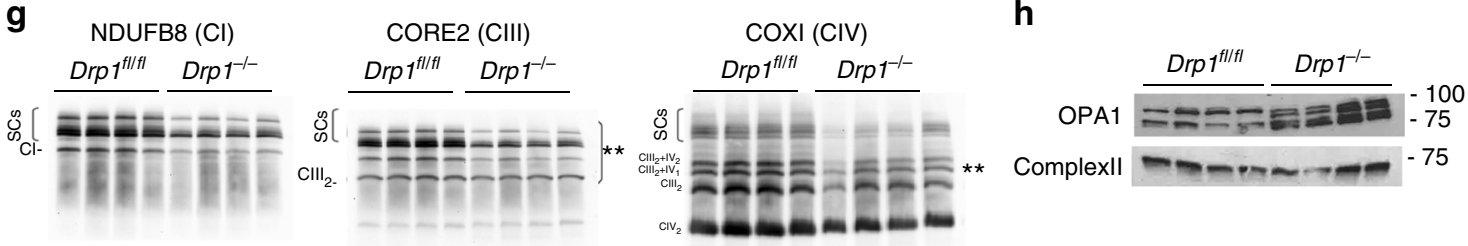

Fig. 2 Muscle DRP1-chronic inhibition alters mitochondrial morphology, RCS organization and complex-I- and complex-II-dependent respiration. a Representative EM cross-sectional images of mitochondrial distribution and morphology in gastrocnemius from WT and Drp1-/- mice. In cross-sections, the different morphology of mitochondria in Drp1 ${ }^{-/-}$from WT is often evident: in Drp1 ${ }^{-/-}$fibers mitochondria are more often larger in size (scale bar: $1 \mu \mathrm{m})$. $\mathbf{b}$ Mitochondrial DNA copy number quantification. mtDNA was amplified by RT-PCR from the total DNA of gastrocnemius muscles of the indicated genotype. The data are normalized to control and represent average \pm SEM of five independent experiments. c Representative immunoblots of four independent experiments of muscle homogenates showing no difference of porin/GAPDH ratio. d RT-PCR analysis of mitochondria dynamics transcripts of muscles of WT and Drp1-/-. DRP1 deletion induces PGC1 $\alpha$, OPA1, and Mfn1 transcript levels. The data represent average \pm SEM ( $n=8)$ and are normalized for GAPDH and expressed as fold increase of controls. e Succinate dehydrogenase (SDH) staining of Drp $f^{f / / f l}$ and Drp1 ${ }^{-/-}$muscles show a different distribution of mitochondrial network in $\mathrm{KO}$ mice. $\mathbf{f}$ Respiratory control ratio (RCR) of muscle-isolated mitochondria energized with $5 \mathrm{mM} / 2.5 \mathrm{mM}$ GLU/MAL or $10 \mathrm{mM}$ SUCC. The data represent average \pm SEM of three independent experiments. $\mathbf{g}$ DRP1 deficiency leads to a significant reduction of CIII and CIV assembly in respiratory chain supercomplexes (RCS). Representative Blue Native PAGE analysis showing RCS developed and normalized for individual respiratory chain complexes. Left panel: CI subunit NDUFB8, middle panel: CIII-Core2 protein 2 and right panel: CIV subunit COXI. h Representative immunoblot analysis of OPA1 different isoforms show an increase in OPA1 cleavage in Drp1-null muscles $(n=4)$. Two-tailed unpaired Student's $t$ test was used. Statistical significance: ${ }^{\star} p \leq 0.05 ;{ }^{\star \star} p \leq 0.01 ;{ }^{\star \star \star} p \leq 0.001$

postnatal growth. IGF1 transcript level was downregulated in hepatocytes (Fig. 3h), and consistently, the IGF1 blood level was also reduced in knockout mice (Fig. 3i). The decrease of IGF1 explains the reduced size of DRP1-deficient mice.

Since the size of the cell is determined by the balance between protein synthesis and degradation, we also monitored the atrophy-related pathways ${ }^{26,27}$. Protein breakdown is controlled by a transcriptional-dependent program that regulates the expression of a subset of genes named atrophy-related genes. These genes encode for enzymes that catalyze rate-limiting steps during ubiquitination process and autophagosome formation. FoxO3 is the master regulator of the atrophy program, and its activity is controlled at multiple levels, including its transcription. Interestingly, FoxO3 was induced in Drp1 $1^{-1-}$ muscles (Fig. 3j) and its downstream targets, the atrophy-related ubiquitin ligase atrogin-1 and MuRF1, were upregulated (Fig. 3k). We also measured a new set of ubiquitin ligases that we have identified to be under FoxO regulation and involved in muscle atrophy ${ }^{26,28}$. Among them, MUSA1 was upregulated in Drp1 $1^{-1-}$ mice (Fig. 31). According to the induction of the ubiquitin ligases, also the amount of lysine 48 and lysine 63 polyubiquitinated proteins was increased in Drp1 $1^{-1-}$ mice (Fig. $3 \mathrm{~m}$ ). Since the autophagylysosome system is also induced during muscle atrophy and is controlled by FoxO3 ${ }^{29}$, we measured expression of FoxOdependent autophagy-related genes ${ }^{26}$. Several genes were induced in knockout mice (Fig. 3n) confirming that an atrophy program was activated in these mice. However, the ratio of LC3II on GAPDH was only mildly increased, while p62 was not affected (Fig. 3o; Supplementary Fig. 3b), suggesting that autophagy was weakly induced at 12 days of postnatal life. In conclusion, DRP1 deletion led to a defect of muscle growth caused by a fasting-like condition, inhibition of protein synthesis, and mild induction of the ubiquitin-proteasome and autophagy-lysosome-dependent protein breakdown. 
a<smiles>[CH]C([CH-])C</smiles>

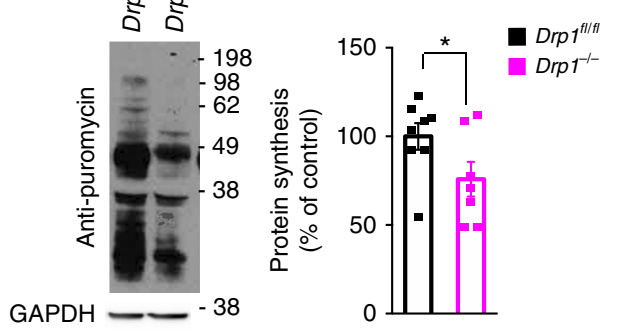

b
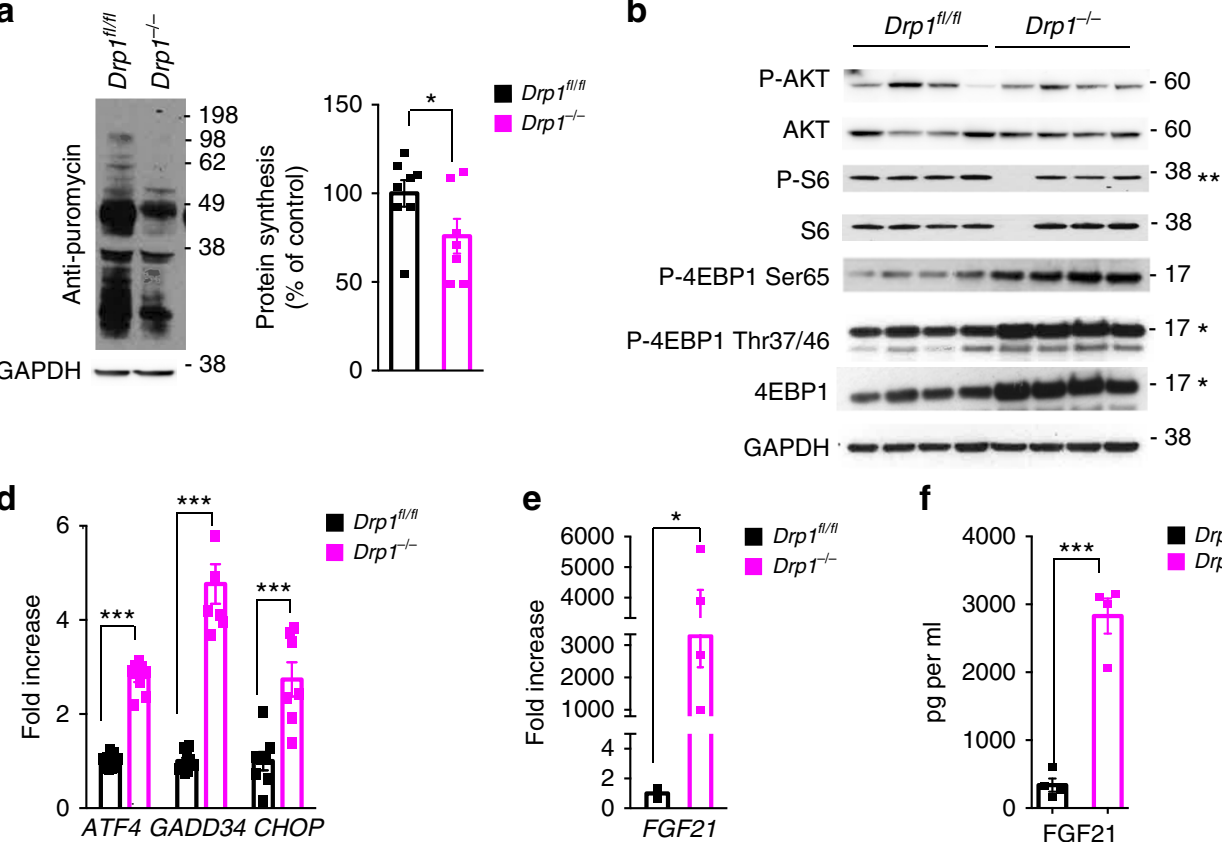

e

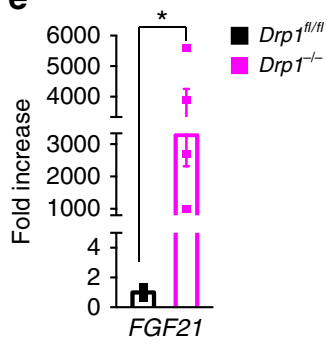

f

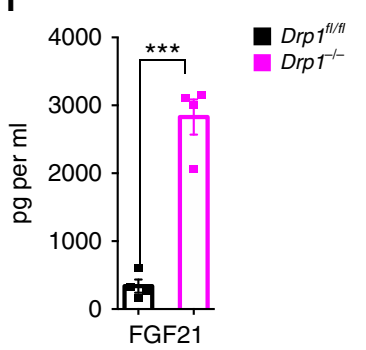

C

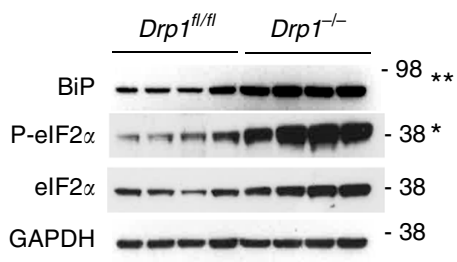

h

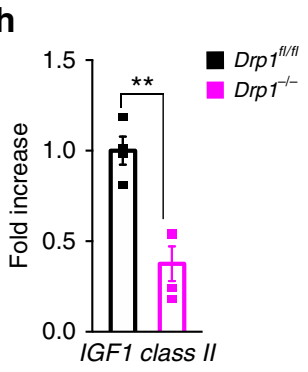

I

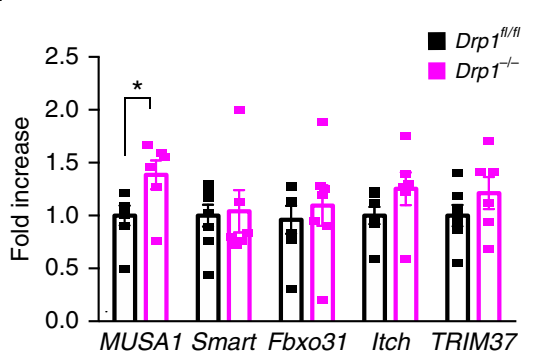

i

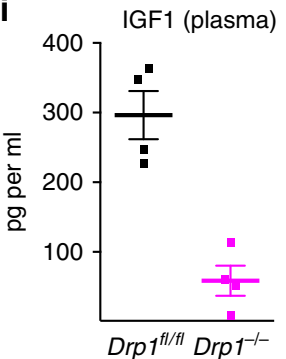

m $\mathrm{j}$

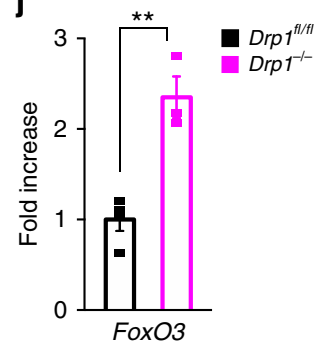

k
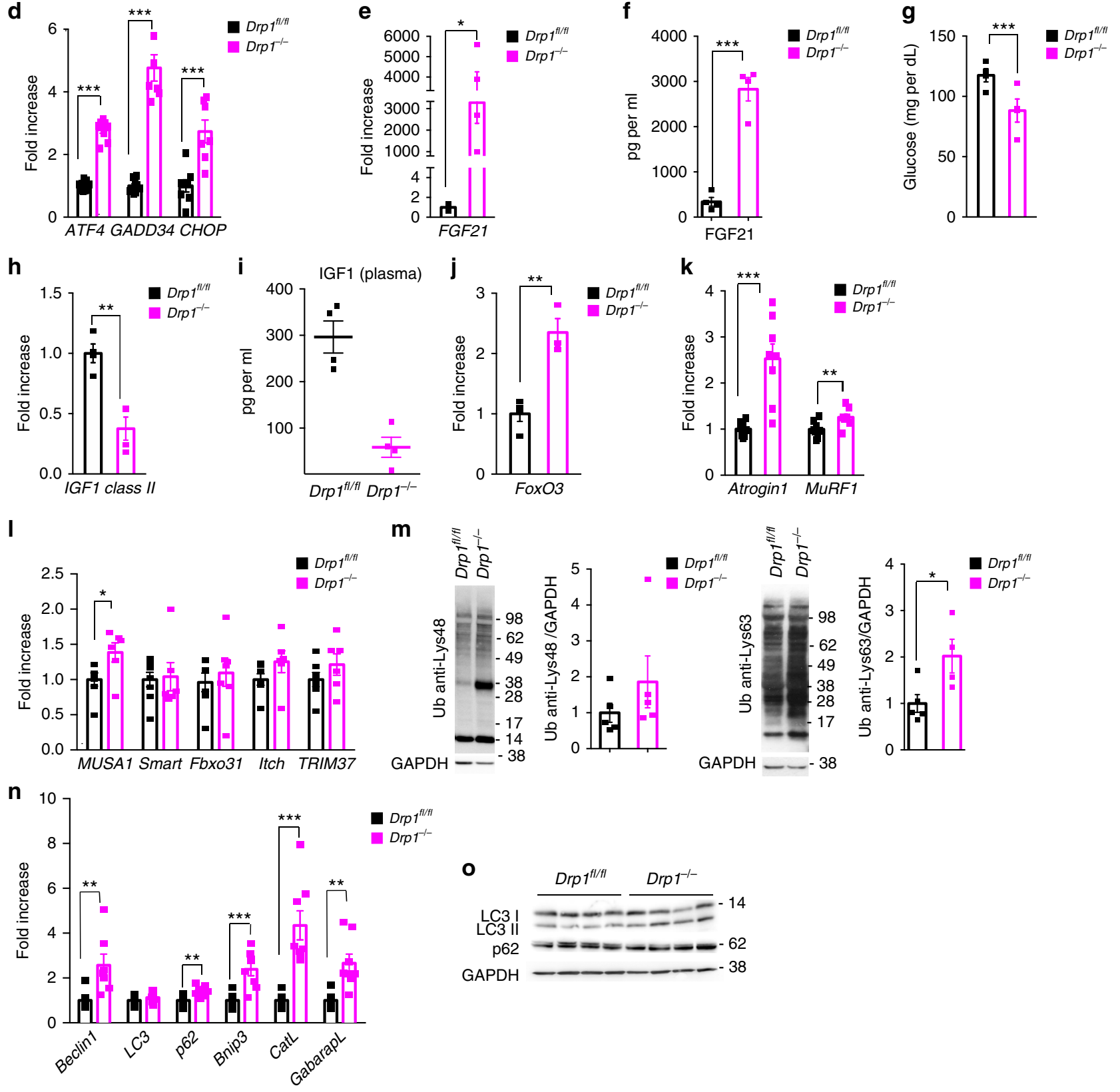

Acute deletion of Drp1 causes muscle wasting and weakness. The severe phenotype of $D r p 1^{-1-}$ mice hindered further analyses in adulthood. We therefore generated a tamoxifen-inducible muscle-specific Drp1 knockout mouse $\left(D r p 1^{-/-}\right)$by crossing $D r p 1^{\mathrm{fl} / \mathrm{fl}}$ mice with HSA-Cre-ER transgenic mice. Tamoxifen treatment in 5-month-old mice efficiently reduced DRP1 protein in different muscles (Fig. 4a). Within 50 days from tamoxifen treatment, mice started to differ in body weight when compared with controls (Fig. 4b). The reduction of body weight was secondary to important decrease of muscle mass. Skeletal muscles 
Fig. 3 DRP1 absence leads to downregulation of protein synthesis and activation of the Ubiquitin-proteasome and autophagy-lysosome pathways. a In vivo SUnSET technique showed a significant reduction of protein synthesis in DRP1-ablated mice muscles. Representative western blot and quantification of the puromycin-labeled peptides, expressed as percentage of the values obtained in the control group, are depicted. The data represent average \pm SEM of eight independent experiments. b The total protein extracts from newborns muscles were immunoblotted with the indicated antibodies. A representative immunoblot of four independent experiments is shown. Statistical significance of specified densitometric ratios is indicated on the right. The data are normalized to GAPDH $(n=8)$. Representative immunoblot $(\mathbf{c})$ and RT-PCR $(\mathbf{d})$ showing the activation of the unfolding protein response (UPR) pathway in DRP1 KO muscles. The data represent average \pm SEM of seven independent experiments. e RT-PCR analysis of transcriptional levels of muscle FGF21 $(n=3)$. $\mathbf{f}$ Quantification of blood levels of FGF21 shows an increase in DRP1 KO mice $(n=3)$, leading to a decrease in blood glucose $(\mathbf{g}, n=4)$ and to a significant decrease in IGF1 class II mRNA level in the liver $(\mathbf{h}, n=4)$. The data represent average \pm SEM. $\mathbf{i}$ Quantification of IGF1 in plasma from Drp1-/and control mice shows a significant decrease of IGF1 circulating levels in the absence of DRP1. RT-PCR analysis of FoxO-dependent transcripts shows an increase in FoxO3 levels (j), in the muscle-specific ubiquitin ligases Atrogin1 and MuRF1 (k) and in the novel Ubiquitin-ligase MUSA1 (I). The data represent average \pm SEM ( $n=6$ per condition). $\mathbf{m}$ Representative blots and densitometric analysis of at least four independent experiments of total muscle extracts immunoblotted for anti-Ubiquitin (Lys48) and for anti-Ubiquitin (Lys63) normalized to GAPDH. The data represent average \pm SEM. $\mathbf{n}$ Quantitative PCR analysis of autophagy-related transcripts showing a significant induction of autophagy markers in Drp1 ${ }^{-/-}$. The data represent average \pm SEM $(n=8$ per condition). o Representative immunoblots of four independent experiments of autophagy-related proteins. The data represent average \pm SEM $(\mathrm{WT}, n=4 ; \mathrm{KO}, n=6)$. Two-tailed unpaired Student's $t$ test was used. Statistical significance: ${ }^{\star} p \leq 0.05 ;{ }^{* \star} p \leq 0.01 ;{ }^{* \star *} p \leq 0.001$

were smaller (Fig. 4c), and fiber size of $D_{r p 1^{-1}}$ was reduced by 25 and $50 \%$ when compared with controls at 70 and 180 days after the treatment (Fig. 4d). Concomitantly, there is also a decrease of white adipose tissue (Supplementary Fig. 4a). While fiber-type distribution was not affected at 40 days, an increase of $2 \mathrm{X}$ fibers and a concomitant trend of decrease of type $2 \mathrm{~A}$ and $2 \mathrm{~B}$ fibers were detected at 70 days (Supplementary Fig. $4 \mathrm{~b}$ ). Morphological analyses showed pure atrophy with no features of myofiber degeneration at 70 days (Fig. 4e). The decrease of fiber size was detected in all the different fiber types (Supplementary Fig. 4c, d). Moreover, muscle loss in DRP1-deficient mice was enhanced when nutrients were removed for $24 \mathrm{~h}$, a time that does not induce significant myofiber atrophy in control mice (Supplementary Fig. 4e). Therefore, inhibition of DRP1 sensitizes muscle cells to the atrophy program. However, prolonged DRP1 deletion caused myofiber death as revealed by the appearance of center-nucleated fibers (Fig. 4e, f). This myopathic phenotype is evident at 180 days, but it appears at 120 days from treatment. Quantification of center-nucleated fibers confirmed that degeneration followed by regeneration happened in 10 and $25 \%$ of myofibers in TA and gastrocnemius muscles, respectively (Fig. 4f). To understand whether muscle performance was affected, we measured the strength of gastrocnemius muscle in living mice at 70 days after tamoxifen treatment when muscles display pure atrophy and do not show center-nucleated fibers. Single twitch and maximal absolute force were reduced (Fig. 4g), suggesting a loss of contractile proteins. However, the specific force, the force normalized for muscle mass, was also decreased in knockout mice (Fig. 4g). This finding suggests that, besides a decrease of myosin-actin content, there is also an impairment in force generation. We also measured muscle force and fatigue, ex vivo, in two other different muscles, EDL and Soleus. Interestingly, while muscle force was decreased during twitch and tetanic contractions in EDL, at 70 days, and in Soleus, already at 40 days after treatment, the fatigue was improved in EDL and Soleus of DRP1-deficient mice (Supplementary Fig. 5a, b). This apparent paradox could be explained by the lower force developed by DRP1-deficient muscles compared with control muscles and consequently, less ATP consumption during contraction. Thus, acute inhibition of DRP1 causes muscle loss and important weakness, but ameliorates fatigue.

Acute deletion of Drp1 causes mitochondrial dysfunction. SDH staining was normal, but size of the puncta in oxidative fibers was increased, suggesting that mitochondria were bigger (Fig. 5a). Analyses of the mitochondrial ultrastructure by EM confirmed the presence of morphological modifications in $\mathrm{Drp1}^{-1-}$ fibers compared with controls. In $\operatorname{Drp} 1^{\mathrm{f} / \mathrm{fl}}$ EDL fibers (left panel of Fig. 5b), mitochondria are usually placed at the I band in proximity of $\mathrm{Z}$ lines (pointed by arrowheads), next to the calcium release units (CRUs) ${ }^{30}$. Healthy mitochondria usually exhibit an electron-dense matrix, with parallel internal cristae (Fig. 5b, inset). In $\operatorname{Drp}^{-1-}$ fibers, about $10 \%$ of mitochondria are damaged (versus only $2 \%$ in WT), larger in the size (white arrowhead and inset in the center panel of Fig. 5b), and often longitudinally oriented between myofibrils (the center panel of Fig. 5b; large arrows). Moreover, the relative fiber volume occupied by the mitochondria is increased when compared with WT, even if their number is reduced (Supplementary Table 1). Finally, about $20 \%$ of fibers from $\operatorname{Drp1}^{-1-}$ muscles contains spindleshaped structures, which are surrounded by a membrane and contains vacuoles, possibly representing degeneration of large swollen mitochondria (right panel of Fig. 5b, asterisks). Consistent with the morphological abnormalities, myofibers from tamoxifen-treated $\operatorname{Drp}^{-1-}$ flexor digitorum brevis (FDB) muscle showed an increased incidence of the mitochondria that depolarized when the $\mathrm{F}_{1} \mathrm{~F}_{0}$-ATPase was blocked by oligomycin ${ }^{31}$ (Fig. 5c). Moreover, TMRM staining revealed the area of the myofiber where mitochondria were completely depolarized (Fig. 5c). Accordingly, mitochondrial respiration (Fig. 5d; Supplementary Fig. 6a) was reduced, while complex activity is not greatly affected (Supplementary Fig. 6b). We also revealed a general reduction of the subunits of the different respiratory chain complexes, being complex-I the most affected (Supplementary Fig. 6c), and of supercomplex assembly (Fig. 5e; Supplementary Fig. 6d). Similar to the conditional knockout, the mitochondrial DNA content and mitochondrial mass revealed by TOM20 and porin did not differ between the two genotypes (Fig. 5f, g; Supplementary Fig. 6d). However, despite the alteration in respiration, mitochondrial ROS production was not increased by DRP1 ablation (Supplementary Fig. 6e). In conclusion, Drp1 ablation in adult animals alters mitochondrial morphology and function independently of mtDNA content and mitochondrial mass.

Acute deletion of Drp1 induces ER stress and blocks autophagy. Similar to the results obtained in conditional Drp1-deficient mice, the Akt-mTOR axis appeared inconsistent with the atrophic phenotype being 4EBP1 robustly hyper-phosphorylated in two mTOR-dependent sites and S6 unchanged (Fig. 6a; Supplementary Fig. 7a). When we checked protein synthesis, in vivo, we did not find any difference (Fig. 6b; Supplementary Fig. 7b). Consistent with the conditional knockout, we found an activation of the UPR pathway. Bip/Grp78 and p-eIF2a (Fig. 6c; 
a TA

SOLEUS
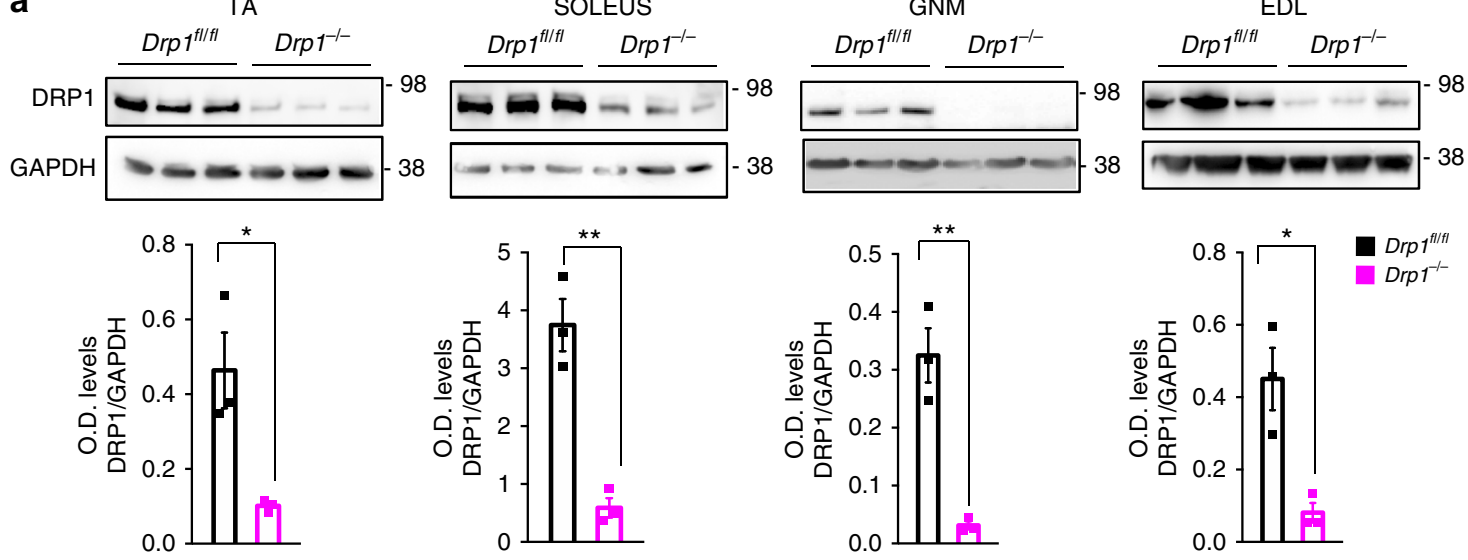

b

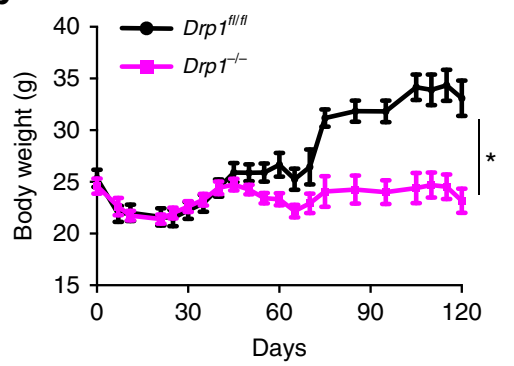

C
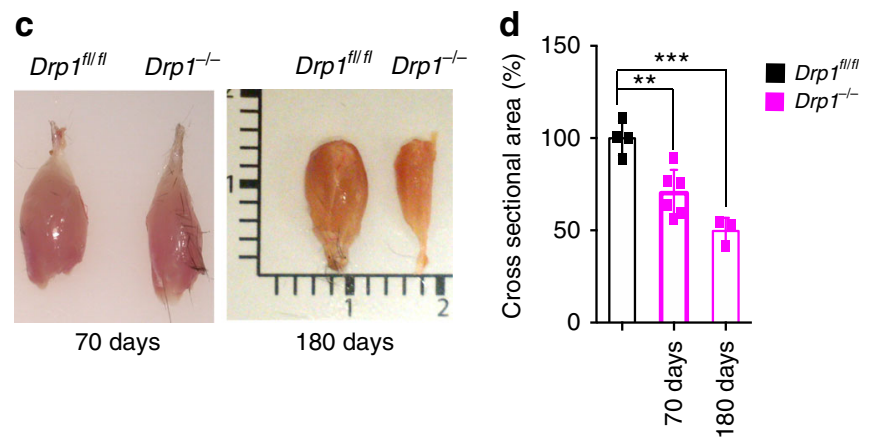

e
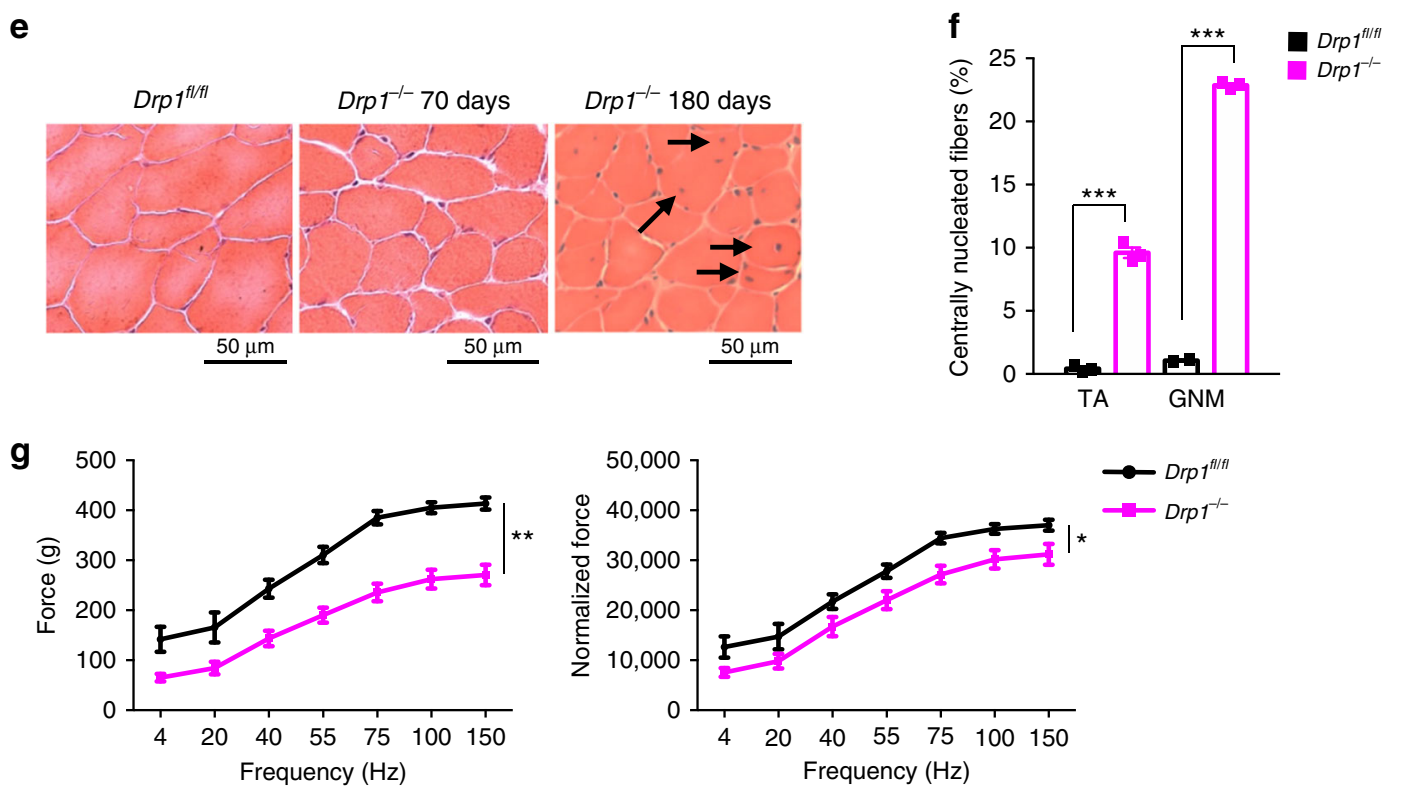

Fig. 4 DRP1 loss in adult animals causes body weight loss, muscle atrophy and muscle weakness. a DRP1 protein levels are downregulated in different types of skeletal muscles in HSADRP1-null mice. O.D. levels represent the average of at least three independent experiments \pm SEM. $\mathbf{b}$ Growth curve of control and $\mathrm{Drp}^{-1}{ }^{-1}$ littermates during and after tamoxifen treatment. KO mice start to lose body weight after 7 weeks of tamoxifen treatment and during the following weeks. The data represent average \pm SEM (WT, $n=10 ; \mathrm{KO}, n=11)$. c Dissected gastrocnemius muscles from the control and DRP1-null mice show an important muscle atrophy after DRP1 deletion in adult animals. $\mathbf{d}$ Quantification of the cross-sectional area of myofibers indicates a significant reduction in DRP1-ablated muscles. Values represent average \pm SEM (WT, $n=5 ; \mathrm{KO} 70$ days, $n=5 ; \mathrm{KO} 180$ days, $n=3$ ). e Representative haematoxilin-eosin staining of Tibialis Anterior muscle showing signs of myofiber degeneration/regeneration in Drp1-/- after 180 days of treatment. f In $\mathrm{Drp}^{-/-}$muscles after 180 days of treatment, there are 10 and $25 \%$ of myofibers are centrally nucleated, in tibialis anterior muscle and gastrocnemius muscle, respectively ( $n=3$ mice each condition). $\mathbf{g}$ Force measurements performed in vivo on gastrocnemius muscles. Absence of DRP1 leads to a significant decrease in both absolute force and maximal specific force generated during tetanic contraction. The data represent average \pm SEM $(n=3)$. Two-tailed unpaired Student's $t$ test and two-way analysis of variant (ANOVA) were used. Statistical significance: ${ }^{\star} p \leq 0.05$; ${ }^{\star \star} p \leq 0.01$; ${ }^{\star * \star} p \leq 0.001$ 
a

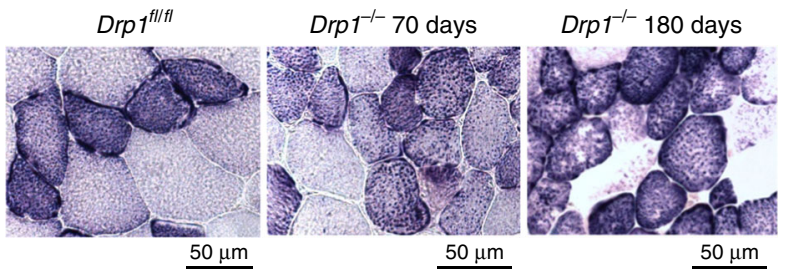

b

$\operatorname{Drp}^{f|/| f \mid}$

$\operatorname{Drp}^{-1-}$
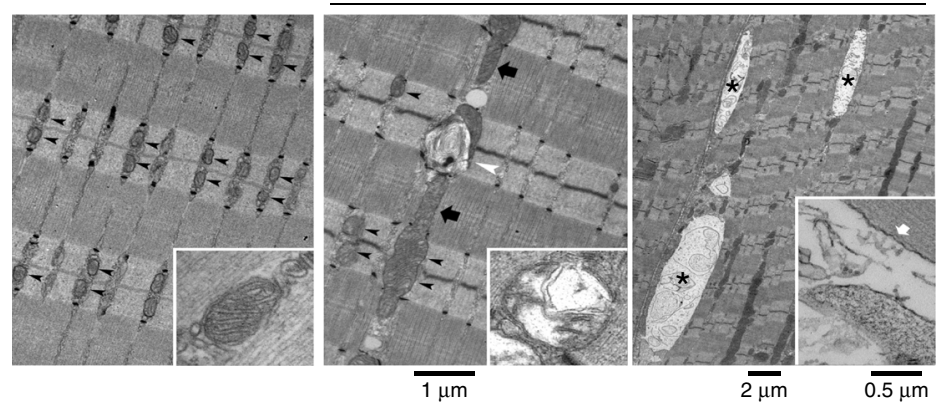

C

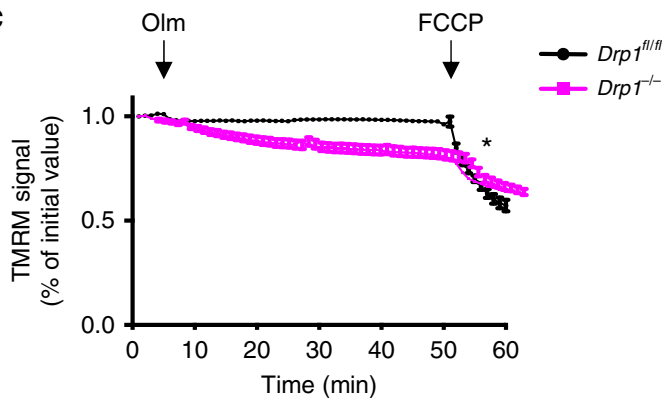

d

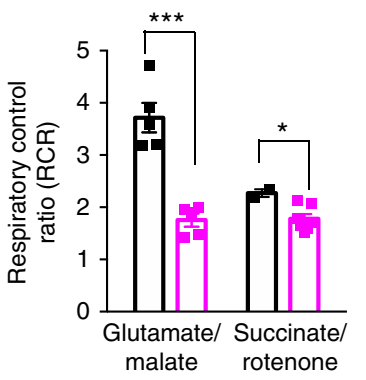

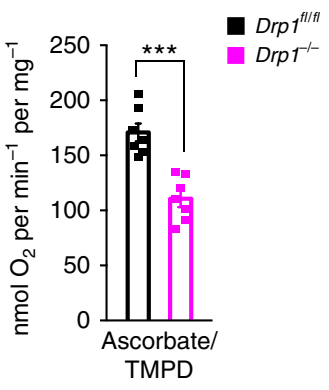
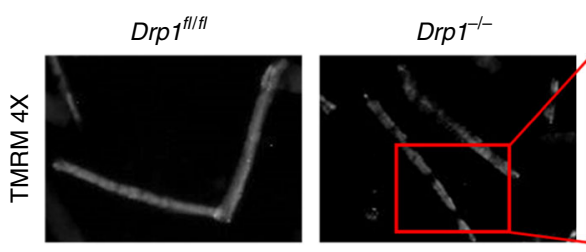

e
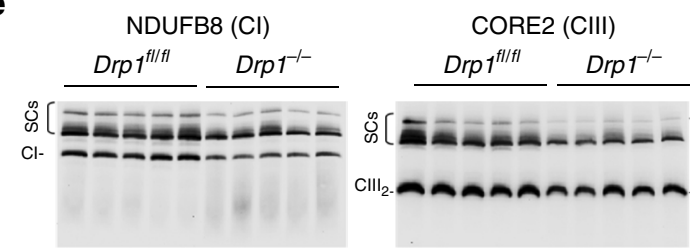

9

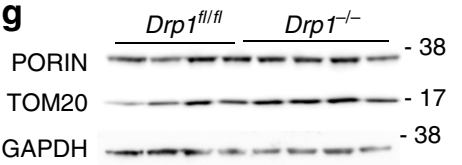

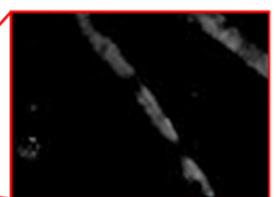
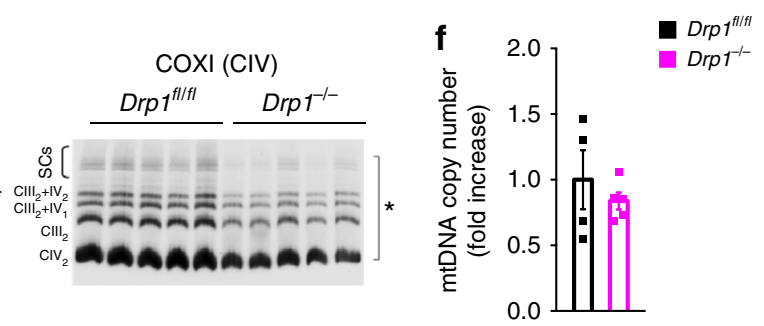

Supplementary Fig. 7a) and the transcripts of ATF4 and downstream genes, including FGF21, were induced in DRP1-null muscles (Fig. 6d; Supplementary Fig. 7c). FGF21 blood levels were also increased of 20 -fold in DRP1 knockout mice when compared with controls (Supplementary Fig. 7d). FoxO3 is the master regulator of the atrophy program and its activity is controlled at the transcriptional level and by multiple pathways via post-translational modifications, which include phosphorylation, acetylation, oxidation, and methylation ${ }^{26,32}$. Therefore, we monitored the presence of FoxO3 in the nuclear fraction as a readout of its activity and found an increased nuclear translocation in DRP1-deficient mice (Fig. 6e). The increase of the total 4EBP1 is also consistent with FoxO activation, being 4EBP1 a downstream target. Then we checked the status of the ubiquitin-proteasome and autophagy-lysosome systems. Interestingly, while atrogin 1 and $M u R F 1$ did not change, other two FoxO-dependent ubiquitin ligases MUSA1 $1^{28,33}$ and Itch were upregulated in DRP1-deficient muscles (Fig. 6f). We then monitored the FoxO-dependent autophagy-related genes and we found an upregulation of most of these genes (Fig. $6 \mathrm{~g}$ ).

However, when we checked autophagosome formation, we did not detect an increase of lipidated LC3II, but instead we found an 
Fig. 5 Ablation of DRP1 in adulthood modifies mitochondrial shape and reduces RCS assembly, Complex-I-, Complex-II-, and complex-IV-dependent respiration. a SDH staining indicating the presence of bigger mitochondria in $\mathrm{KO}$ muscles compared with control. b Representative electron micrographs of EDL muscles of controls and Drp1-/-. Left panel: in WT mitochondria (pointed by black arrowheads) are placed in proximity of Z lines and usually exhibit an electron-dense matrix (inset). Center panel: in Drp1-/- mice mitochondria are often larger in size, oriented longitudinally (large arrows), and damaged (white arrowhead and inset). Right panel: a Drp1-/- fiber presenting spindle-shaped regions (asterisks) which are surrounded by a membrane (inset, white arrow) and that contains vacuoles. c Absence of DRP1 induces mitochondrial depolarization. Isolated adult fibers were loaded with TMRM. Oligomycin and the protonophore FCCP were added at the indicated time points. TMRM staining was monitored in 18 fibers for WT, 52 fibers for KO. Lower panel: representative images of adult myofibers showing altered mitochondrial distribution in DRP1-null muscles. Myofibers were loaded with the potentiometric die TMRM. d Respiratory control ratio (RCR) of muscle isolated mitochondria energized with $5 \mathrm{mM} / 2.5 \mathrm{mM} \mathrm{GLU} / \mathrm{MAL}$ or $2 \mathrm{mM}$ rotenone/10 mM succinate or $3 \mathrm{mM}$ ascorbate/10 mM TMPD. The data represent average \pm SEM (GLUT/MAL, $n=5$ each condition; succinate/rotenone: WT, $n=2 ; \mathrm{KO}$, $n=7$; ascorbate/TMPD, $n=7$ each condition). e The assembly of CIII and CIV in RCS is significantly reduced in mitochondria of DRP1-deficient muscles. Representative Blue Native PAGE analysis showing RCS developed and normalized for individual respiratory chain complexes. CI subunit NDUFB8 (left panel), CIII-Core2 protein 2 (middle panel), and CIV subunit COXI (right panel). f Mitochondrial DNA copy number quantification in controls and $\mathrm{KO}$ muscles. mtDNA was amplified by RT-PCR from the total DNA of gastrocnemius muscles of the indicated genotype. The data are normalized to controls and represent the average \pm SEM $(W T, n=4 ; K O, n=5)$. $\mathbf{g}$ Mitochondrial mass revealed by TOM20 and porin was not affected by DRP1 deletion $(n=4)$. Two-tailed unpaired Student's $t$ test was used. Statistical significance: ${ }^{\star} p \leq 0.05 ;{ }^{\star \star \star} p \leq 0.001$

accumulation of LC3I, suggesting an autophagy impairment (Fig. 6h; Supplementary Fig. 7a). Next, we tested autophagy flux and we found no increase of p62 and LC3II upon colchicine treatment in DRP1-deficient mice (Fig. 6i). These findings confirm that autophagy is inhibited. Since mitochondrial fragmentation is blocked, we tested whether mitophagy is also impaired. Adult muscles were transfected with a $\mathrm{pH}$-sensitive mitochondrial targeted probe that changes the fluorescence upon medium acidification (mitoKeima). Therefore, this probe reflects the autophagy-dependent delivery of the mitochondria to lysosome (mitophagy). The measurements confirmed that mitophagy is reduced in DRP1-deficient muscles (Fig. 6j). In conclusion, loss of DRP1 in adult muscle is sufficient to induce a catabolic condition that involves activation of the UPS system and inhibition of autophagy and mitophagy pathways ${ }^{34}$.

Inhibition of DRP1 alters normal calcium homeostasis. Acute deletion of DRP1 causes weakness, myofiber degeneration, ER stress, and appearance of the swollen/dysfunctional mitochondria. All these features can be triggered by disruption of $\mathrm{Ca}^{2+}$ homeostasis. Therefore, we initially measured cytosolic calcium of Drp $1^{-1-}$ and controls in basal condition and found no difference (Fig. 7a). However, when we stimulated the $\mathrm{Ca}^{2+}$ release from the sarcoplasmic reticulum (SR), either by electrical stimulation or by caffeine/thapsigargin treatment, we showed a reduction of the cytosolic $\mathrm{Ca}^{2+}$ transient in DRP1-deficient myofibers (Fig. 7b, c; Supplementary Fig. 8a, b). These changes occurred early after 40 days from tamoxifen treatment when animals have not yet lost muscle mass and progressively increased at 70 days.

To test whether $\mathrm{Ca}^{2+}$ store in SR was reduced, we checked the concentration of this ion by transfecting adult muscle with SRtargeted cameleon probes. However, no differences were found between DRP1 knockout and controls, either in basal or after electrical stimulation (Supplementary Fig. 8c, d, g). Importantly, the changes of $\mathrm{Ca}^{2+}$ concentration in the SR after electrical stimulation did not differ between controls and DRP1-deficient mice, suggesting that the two genotypes release the same amount of $\mathrm{Ca}^{2+}$ (Supplementary Fig. 8g). Consistently, calsequestrin, the most important $\mathrm{Ca}^{2+}$ buffering protein in the SR, did not change between knockout and controls (Supplementary Fig. 9c). We then measured free $\mathrm{Ca}^{2+}$ concentration in the mitochondrial matrix by using a specific cameleon probe and we found that the peak of $\mathrm{Ca}^{2+}$ concentration reached during high-frequency electrical stimulation was higher in DRP1-deficient myofibers (Fig. 7d; Supplementary Fig. 8e, f). This suggests that the presence of an active and not depolarized mitochondrial population was able to uptake $\mathrm{Ca}^{2+}$ from the cytosol.
Importantly, when we checked the level of the mitochondrial calcium uniporter (MCU), the mitochondrial channel for $\mathrm{Ca}^{2+}$ uptake, we showed an increase of MCU in mitochondria of DRP1-deficient mice (Fig. 7e; Supplementary Fig. 9a). This increase is not consequent to transcription because the $M C U$ transcripts did not differ between control and DRP1 knockout (Supplementary Fig. 9b). We have recently shown that MCU translation is controlled by $\mathrm{miR} 1^{35}$. When we monitored miR1 expression, we found that it was downregulated in DRP1deficient muscles when compared with controls (Fig. 7f). Therefore, DRP1 inhibition causes increased MCU expression via miR1 downregulation and enhanced $\mathrm{Ca}^{2+}$ uptake of the mitochondria.

The reduction of cytosolic $\mathrm{Ca}^{2+}$ upon stimulation could, in principle, also result from modifications of CRUs, the structures deputed to excitation contraction (EC) coupling. To address this point, we performed EM analyses and found some modification in CRUs compared with WT fibers (Fig. 7g). Indeed, we showed an increased frequency of oblique/longitudinal triads (inserts D and E) and a decrease in the number/area of CRUs compared with WT fibers (Fig. 7g, inserts D-F; Supplementary Table 2, column A). The combined reduction in the number of the mitochondria (Supplementary Table 1, column B) and of CRUs (Supplementary Table 2, column A), results in a reduced number of mitochondria-CRUs pairs (Supplementary Table 2, column D). When we checked the expression level of the SR-mitochondrial tethering protein Mitofusin2 and the ER chaperone, calnexin ${ }^{36}$, we found a downregulation in their expression (Supplementary Fig. 9c). Thus, the impairment of the SR-mitochondria tethering and ER chaperon proteins would explain the abnormalities of CRUs and the presence of ER stress ${ }^{36,37}$.

DRP1 rescue or MCU inhibition normalizes $\mathrm{Ca}^{2+}$ homeostasis. To test the hypothesis that the DRP1-dependent mitochondrial shape is relevant for $\mathrm{Ca}^{2+}$ homeostasis alteration, we performed a rescue experiment. Re-expressing DRP1 protein in DRP1deficient mice enhanced the wave of cytosolic $\mathrm{Ca}^{2+}$ induced by caffeine stimulation. Interestingly, DRP1-mediated mitochondrial fragmentation in control animals also increased the raise of cytosolic $\mathrm{Ca}^{2+}$ induced by caffeine/thapsigargin, supporting the hypothesis that mitochondrial dynamic impacts on $\mathrm{Ca}^{2+}$ homeostasis (Fig. 7h). Importantly, DRP1 overexpression reverted or induced UPR in DRP1 knockout and control mice, respectively (Supplementary Fig. 9d). Finally, to prove that abnormal mitochondrial $\mathrm{Ca}^{2+}$ homeostasis contributes to the phenotype of DRP1 knockout, we knocked down $M C U$ in mice. FDB muscles were transfected by electroporation with expression vectors that encode shRNAs against $M C U$, and 14 days later muscles were 
a

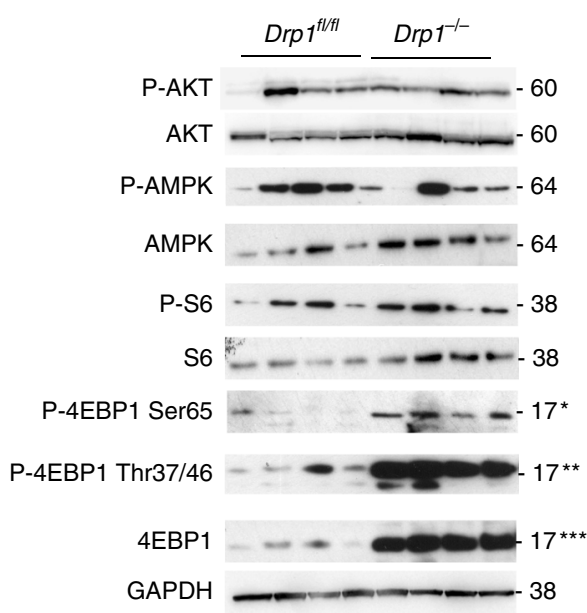

b

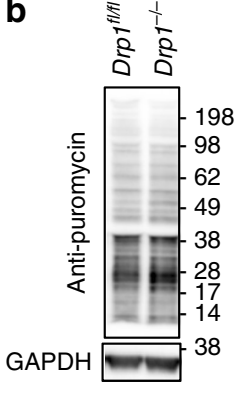

c

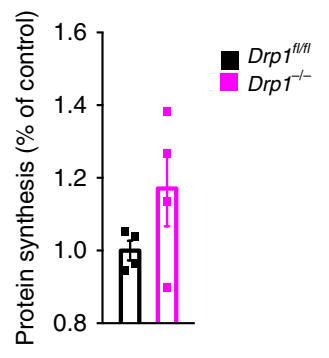

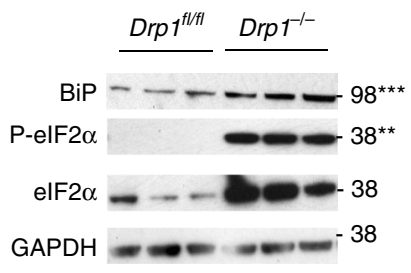
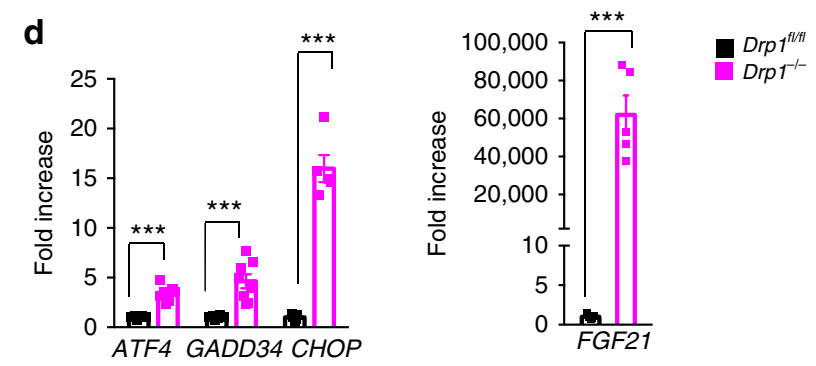

e

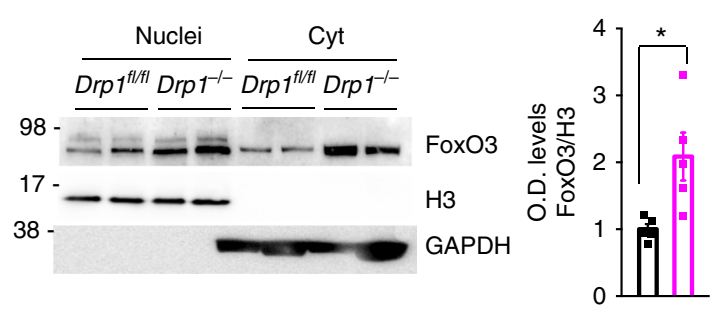

f

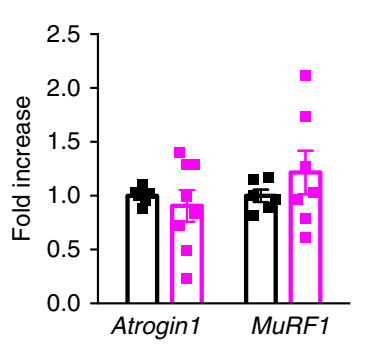

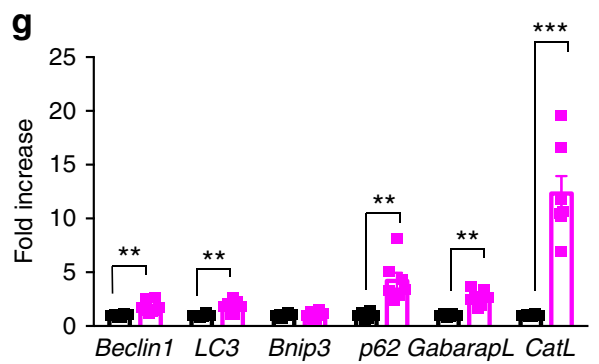

h
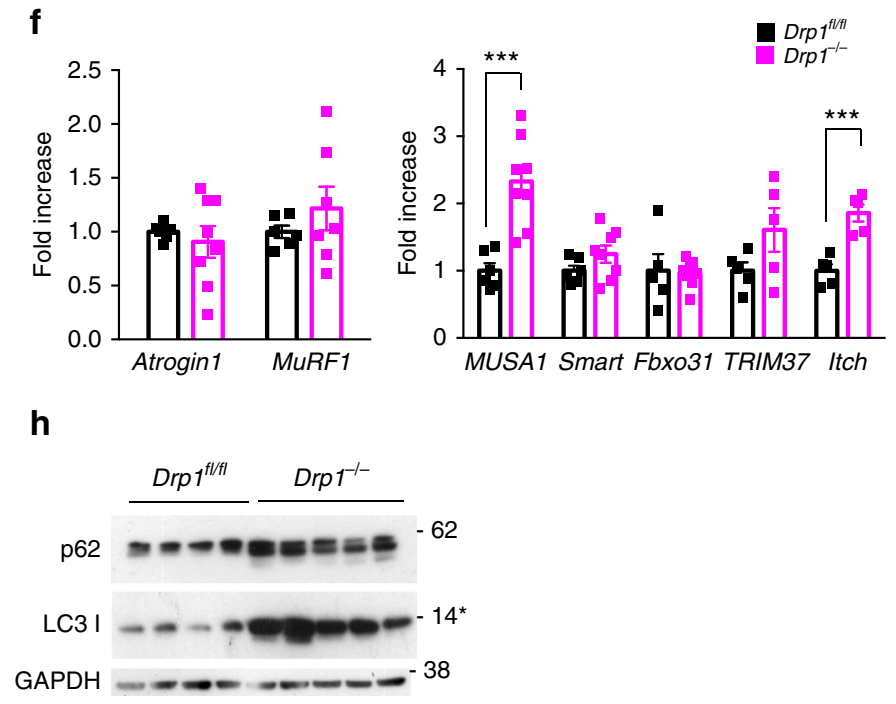

i

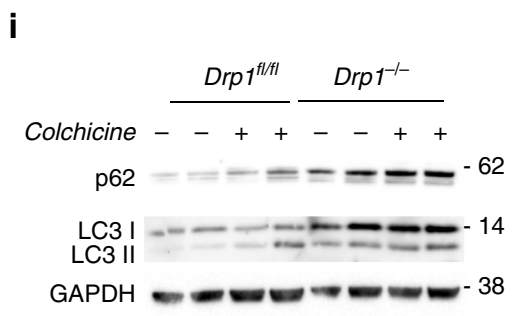

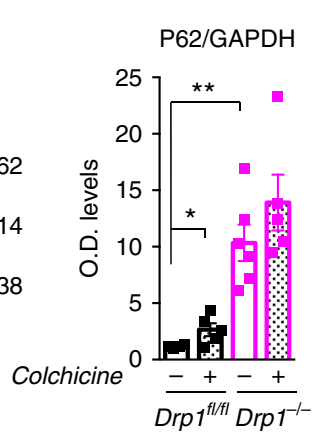

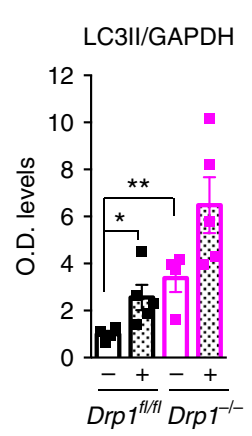

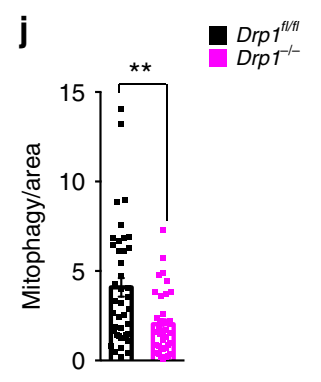

collected, dissected, and analyzed with $\mathrm{Ca}^{2+}$ probe. Inhibition of $M C U$ normalized the amplitude of the wave of cytosolic $\mathrm{Ca}^{2+}$ induced by caffeine stimulation in $\operatorname{Drp1}^{-1-}$ (Fig. 7i). Next, we asked whether by preventing mitochondrial $\mathrm{Ca}^{2+}$ uptake we could ameliorate myofiber survival and ER stress. Muscles were transfected by AAVs with expression vectors for oligos against $M C U$, as previously described ${ }^{38}$. By reducing MCU expression for two months, we reduced the amount of center-nucleated fibers (Fig. 7j) and suppressed UPR (Supplementary Fig. 9e). Altogether, these findings confirm that inhibition of the fission machinery alters the SR-mitochondria tethering, decreases CRU units, increases mitochondrial $\mathrm{Ca}^{2+}$ uptake that, altogether, reduces $\mathrm{Ca}^{2+}$ availability for contraction, and simultaneously induces myofiber death, due to mitochondrial $\mathrm{Ca}^{2+}$ overload. 
Fig. 6 Protein degradation pathways are activated with acute inhibition of DRP1 in adult muscles. a The total protein extracts from adult muscles were immunoblotted with indicated antibodies. A representative immunoblot of four independent experiments is shown. Statistical significance, of specified densitometric ratios is indicated with asterisks on the right. $\mathbf{b}$ In vivo SUnSET technique shows no differences in protein synthesis rate during adulthood $(n=4)$. c Representative immunoblot $(n=4)$ and RT-PCR analysis $(n \geq 5)(\mathbf{d})$ showing the activation of the unfolded protein response and the upregulation of FGF21 in DRP1 KO muscles. e Immunoblot and densitometric quantification showing the accumulation of FoxO3 in the nuclear fraction of tibialis anterior muscles from Drp1 ${ }^{-/-}$mice ( $n=5$ each condition). $\mathbf{f}$ RT-PCR analysis of transcriptional levels of the muscle-specific ubiquitin ligases Atrogin1 and MuRF1 and the novel ubiquitin-ligases MUSA1, SMART, FbxO31, TRIM37, and Itch. The data represent average \pm SEM (at least $n=5$ per condition). $\mathbf{g}$ Quantitative PCR analysis of autophagy-related transcripts showing a significant induction of autophagy markers in Drp1-/- $\mathbf{h}$ Western blot analysis of autophagy marker showing an impaired autophagy in Drp1-/- (WT, $n=4 ; K O, n=5)$. i Immunoblots and relative densitometric quantification from colchicine treated mice support a block in autophagic flux after Drp1 inhibition ( $n \geq 5$ mice per each condition). $\mathbf{j}$ Keima assay indicating the decreased mitophagy in DRP1-KO myofibers (WT, $n=41 ; \mathrm{KO}, n=39$ ). Two-tailed unpaired Student's $t$ test was used. Statistical significance: ${ }^{\star \star} p \leq 0.01 ;{ }^{\star \star \star} p \leq 0.001$

\section{Discussion}

Our study provides insights into the in vivo functions of DRP1 in the muscle of mammals, and therefore sheds light on why DRP1 inhibition causes myopathy in different inherited and acquired diseases ${ }^{13-15,17-20}$. The finding that two different knockouts mice, muscle-specific and inducible muscle-specific, result in striking phenotypes (Supplementary Table 3 ) underlines the critical role of DRP1 in mitochondria function and muscle homeostasis. Like other systems, such as autophagy, both chronic inhibition or acute hyperactivation of mitochondrial fission result in detrimental effects on muscle mass and function, but with different phenotypes and mechanisms. In fact, we and others have shown that overexpression of DRP1 is sufficient to activate an atrophy program via the AMPK-FoxO3 axis ${ }^{10,11}$. However, while the initial outcome of genetic DRP1 ablation is identical to its overexpression, i.e., muscle atrophy via FoxO, our loss-of-function study also reveals the appearance of center-nucleated fibers, a sign of myopathy, and the involvement of different signaling pathways, including MCU upregulation, ER-stress, UPR activation, and great FGF21 induction (Fig. 8). Another peculiarity of the loss-of-function approach is the decrease of the ER-mitochondria tethering protein mitofusin 2 and the down- and upregulation of the ER chaperones calnexin and Bip/Grp78, respectively ${ }^{11}$. Both the decreased mitofusin 2 and calnexin expression explain why ER stress and UPR are induced by DRP1 inhibition ${ }^{36,37}$. ER stress and UPR activation are well-known triggers of FGF21 production in different organs, such as the liver, pancreas, or adipose tissue ${ }^{39}$. Our data show that muscles can be an important source of blood FGF21, when mitochondria are dysfunctional, irrespective of whether this occurs because of a specific mitochondrial dysfunction (as reported here and in ref. ${ }^{13}$ ) or because of mitochondrial dysfunction secondary, for example, to autophagy impairment ${ }^{40}$. The increase of FGF21 can explain the observed metabolic changes, such as basal hypoglycemia, liver GH resistance, and the reduced animal size of conditional knockout mice. However, different phenotypes have been described in mice with high level of FGF21 expression. For instance, FGF21 induction after OPA1 ablation caused systemic inflammatory response and precocious senescence of epithelial tissues such as skin, liver, and gut that resulted in premature death ${ }^{13}$. In DRP1 knockout mice, the main phenotype is not related to ageing but to muscle degeneration and regeneration. The absence of senescence despite the FGF21 induction in DRP1 knockout mice may be explained by the lower level of FGF21 in the serum of DRP1 knockout when compared with OPA1 ${ }^{-1-}$ mice. Alternatively, FGF21 needs to synergize with other factors to elicit the pro-ageing action. Acute deletion of OPA1 in muscles triggers an important IL6 and IL1 upregulation. This induction of inflammatory cytokines is ROS mediated $^{13}$. Interestingly, the inflammatory response is not present and oxidative stress is not increased in DRP1 knockout mice (Supplementary Figs. 9f, 6e). Therefore, the presence of high FGF21 levels associated with high inflammatory cytokines in blood is peculiar of OPA1 knockout mice and may explain the different phenotypes of these inducible muscle-specific knockout mice.

The main peculiarity of DRP1 knockout is an abnormal $\mathrm{Ca}^{2+}$ homeostasis, which is not present in OPA1-deficient mice. These changes may result from modifications of the EC-coupling machinery (Fig. 7g; Supplementary Table 2) but in principle also from the the mitochondria (Figs. 5b, 7d; Supplementary Table 1), which being placed in close proximity to sites of $\mathrm{Ca}^{2+}$ release ${ }^{41}$ are the first to sense the $\mathrm{Ca}^{2+}$ transient via $\mathrm{MCU}^{42,43}$. Importantly, this $\mathrm{Ca}^{2+}$ dysregulation happens before muscle wasting starts, suggesting that it may have a pathogenetic role in DRP1 KO phenotype. The concept that mitochondria affect cytosolic $\mathrm{Ca}^{2+}$ availability is still debated. In fact, the contribution of the mitochondria to cytosolic $\mathrm{Ca}^{2+}$ homeostasis is believed to be dispensable in physiological condition. However, our present data show that the expression of MCU is increased when DRP1 is acutely deleted (Fig. 7e), and that intracellular $\mathrm{Ca}^{2+}$ levels are normalized when MCU was knocked down in vivo (Fig. 7i). Moreover, experiments of DRP1 rescue in knockout and overexpression in control mice (Fig. 7h) support the concept that the mitochondrial network may impact on $\mathrm{Ca}^{2+}$ homeostasis. The increased expression of MCU in $D r p 1^{-/-}$fibers could induce mitochondrial $\mathrm{Ca}^{2+}$ overload, and possibly explains the increased presence of the swollen and damaged mitochondria (Fig. 5 b; Supplementary Table 1). In fact, it is well known that $\mathrm{Ca}^{2+}$ overload triggers permeability transition pore (PTP) opening and mitochondrial swelling ${ }^{44}$. The swelling of mitochondria could, in turn, induce the release of death factors such as endoG, AIF, and cytochrome $\mathrm{c}$ that per se, or in combination with other factors, may cause myofiber death in DRP1-deficient muscles. In fact, when we knocked down MCU, we preserved myofiber survival revealed by the decrease of center-nucleated fibers. We did not completely block the degeneration/regeneration process because the efficiency of transfection was low, and consequently, MCU was reduced only in some myofibers. Interestingly, this scenario is very similar to what happens in Duchenne muscle dystrophy, in which membrane leakage leads to $\mathrm{Ca}^{2+}$ entrance and mitochondria swelling. Indeed, it has been shown that inhibition of PTP opening is beneficial in dystrophic muscles ${ }^{45-47}$.

An apparent surprising finding is the improvement of fatigue in DRP1-deficient mice, despite the presence of a mitochondrial dysfunction. It is important to note that fatigue was measured before the appearance of degenerating/regenerating events. The apparent paradox could be explained by the lower force developed by DRP1-deficient muscles compared with control muscles. The reduction of force due to less contractile proteins and of cytosolic $\mathrm{Ca}^{2+}$ level leads to a decreased ATP consumption during contraction by myosin and SERCA ATPase pump, delaying ATP drop and consequently, improving fatigue. Alternative explanations are also possible: (i) the fiber type switch toward $2 \mathrm{X}$ fiber may contribute to fatigue improvement; (ii) the 
a

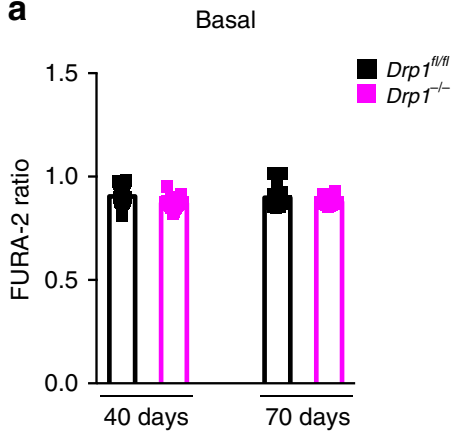

d

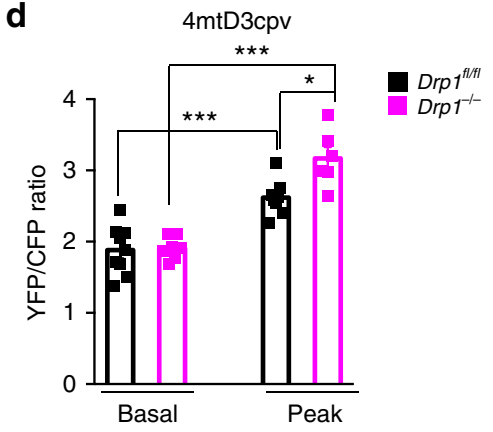

g

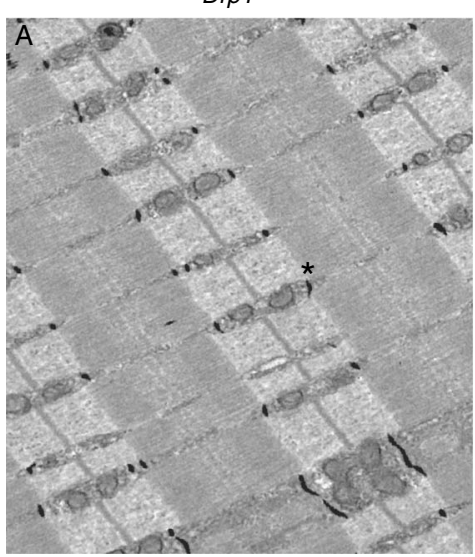

b

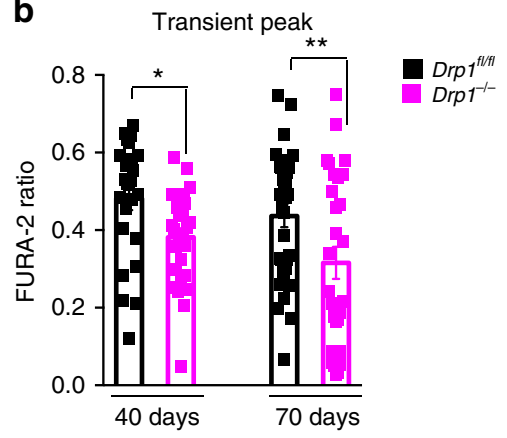

e

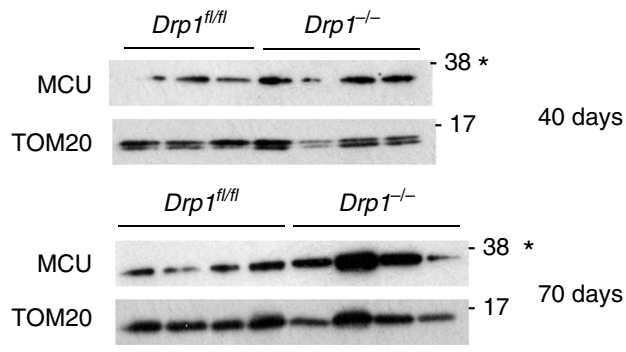

$\operatorname{Drp} 1^{-1-}$

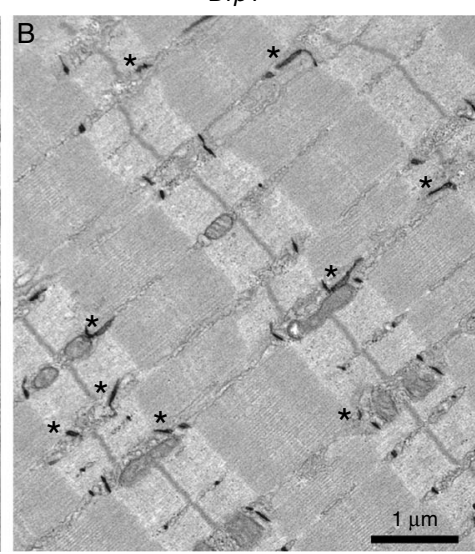

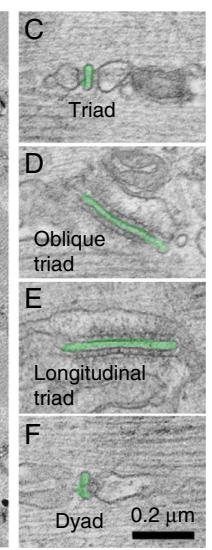

C

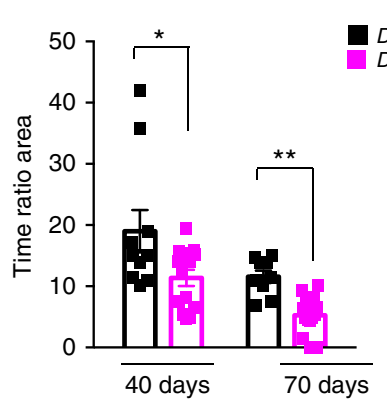

f

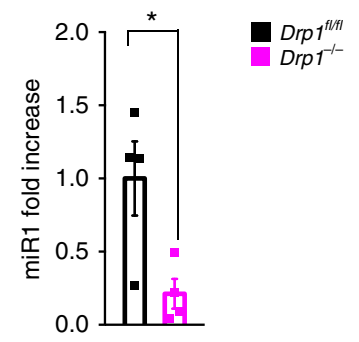
$\operatorname{Drp1}^{f / / f l}$

$\mathbf{h}$

i

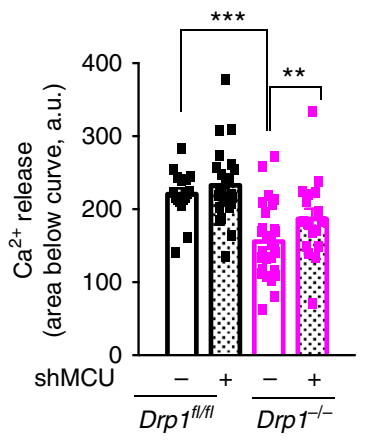

j

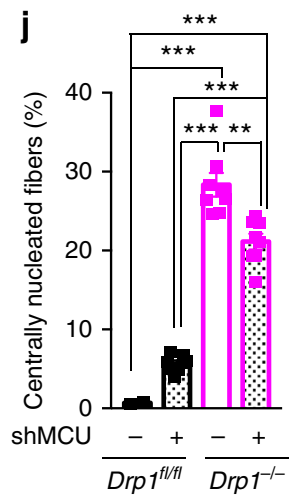

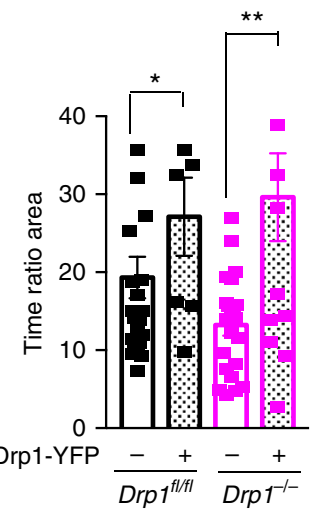


Fig. 7 Acute DRP1 inhibition alters calcium homeostasis. a Cytosolic calcium level at rest is not affected in KO muscles ( 40 days: WT, $n=24 ; \mathrm{KO}, n=28$; 70 days, $n=19$ fibers). b Amplitude of cytosolic $\mathrm{Ca}^{2+}$ transients induced by $0.5 \mathrm{~Hz}$ electrical pulses is lower in $\mathrm{Drp} 1^{-/-}$mice $(40$ days, WT, $n=25 ; \mathrm{KO}$, $n=28$ fibers; 70 days, WT, $n=33 ; \mathrm{KO}, n=29$ fibers). c Cytosolic calcium transients induced by caffeine-thapsigargin are strongly reduced (40 days, WT, $n=10 ; \mathrm{KO}, n=14$ fibers; 70 days, WT, $n=13$ fibers; KO, $n=14$ fibers). d Mitochondrial matrix-free calcium concentration is not affected at rest but is higher in Drp1 ${ }^{-/-}$compared with control during a train of electrical stimulation at $60 \mathrm{~Hz}, 2$ s duration (WT, $n=9 ; \mathrm{KO}, n=7$ fibers; Peak, $n=6$ each condition). e Immunoblot analysis of isolated mitochondria. MCU levels were normalized to TOM2O levels. $\mathbf{f}$ Quantitative RT-PCR analysis showing a significant decrease of miR1 levels in Drp1 ${ }^{-1-}$ skeletal muscle $(n=4$ per condition). The data represent average \pm SEM. $\mathbf{g}$ Ultrastructure and geometry of CRUs in DRP1 KO muscles. Inserts A and B show T-tubule staining in WT (A) and in DRP1 KO (B); the frequency of oblique/longitudinal tubules increases (stars) when compared with control samples. Inserts C-F are representative EM pictures showing the structural geometry of a normal, an oblique, a longitudinal triad and of a dyad respectively. In inserts $\mathbf{A}$ and $\mathbf{B}, \mathrm{T}$-tubules are stained in black while in inserts C-F, T-tubules has been false-labeled in green. $\mathbf{h}$ Cytosolic calcium transients induced by caffeine-thapsigargin after the reintroduction of DRP1 (Drp1-YFP) in muscle fibers. Cytosolic calcium transients in $\mathrm{Drp1}^{-/-}$in the presence of Drp1-YFP show no difference compared with control fibers (Drp $1^{f l / f l}, n=22 ;$ Drp $7^{f l / f l}-$ Drp1-YFP, $n=7$; Drp1 ${ }^{-/-}$, $n=22$; Drp1 ${ }^{-1-}-$ Drp1-YFP, $\left.n=13\right)$. i Inhibition of mitochondria $\mathrm{Ca}^{2+}$ uptake via MCU knockdown restored cytosolic Ca ${ }^{2+}$ levels during caffeine-thapsigargin treatment (WT, $n=17$ fibers; WT shMCU, $n=23$ fibers; KO, $n=27$ fibers; KO shMCU, $n=17$ fibers). j MCU inhibition reduces centrally nucleated fibers in $\mathrm{KO}$ muscles. For all experiments a minimum of three mice was used for each condition. Two-tailed unpaired Student's $t$ test and two-way analysis of variant (ANOVA) were used. ${ }^{\star} p \leq 0.05 ;{ }^{\star \star} p \leq 0.01 ;{ }^{\star \star \star} p \leq 0.001$

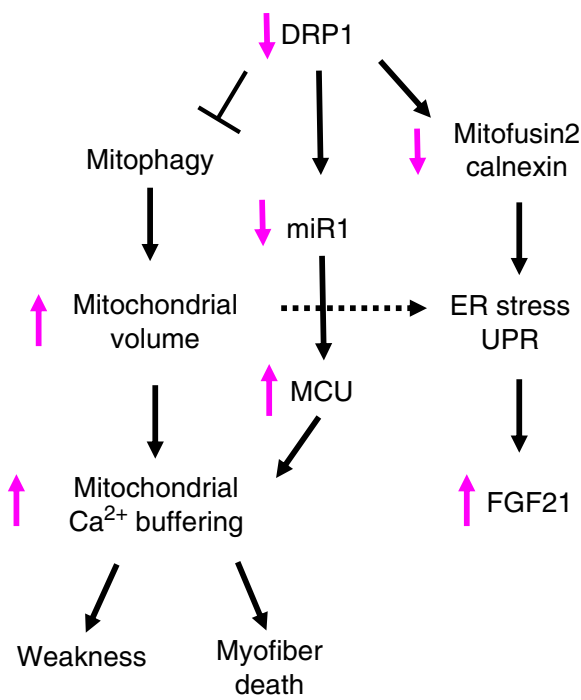

Fig. 8 Scheme of the mechanisms induced by acute DRP1 inhibition

fission deficiency. This finding opens the field to a novel concept in which the shape and spatial organization of mitochondrial network, more than mitochondrial respiration, are sensed by the cell and activate a peculiar biological response. Therefore, the shape of the mitochondria is critical for mitochondrial function, but triggers different signaling pathways and biological processes that are peculiar of fusion or fission impairment.

Finally, therapeutic approaches that maintain a healthy and good shaped mitochondrial network should be considered for the inherited and acquired disorders, characterized by both muscle wasting and mitochondrial dysfunction.

\section{Methods}

Handling and generation of muscle-specific Drp1 null mice. Animals were handled by specialized personnel under the control of inspectors of the Veterinary Service of the Local Sanitary Service (ASL 16 - Padova), the local officers of the Ministry of Health. The use of the animals and the experimental protocol was approved by the ethical committee and by the animal welfare coordinator of the OPBA, University of Padova All procedures are specified in the projects approved by the Italian Ministero Salute, Ufficio VI (authorization numbers 1060/2015 PR), and were conducted in accordance with the relevant codes of practice for the care and use of animals for scientific purposes. Muscles were removed at various time periods and frozen in liquid nitrogen for subsequent analyses.

To generate constitutive muscle-specific Drpl knockout animals, mice bearing $\operatorname{Drp} 1$ floxed alleles $\left(\operatorname{Drp} 1^{\mathrm{fl} / \mathrm{fl}}\right)$ were crossed with transgenic mice expressing Cre under the control of a Myosin Light Chain 1 fast promoter (MLCif-Cre) $)^{21}$.
Experiments were performed on newborns at postnatal day 12. Cre-negative littermates were used as controls.

A second knockout model with inducible muscle-specific deletion of Drp1 was obtained by crossing the $\operatorname{Drp} 1^{\mathrm{f} / \mathrm{fl}}$ line with mice carrying Cre-ER driven by human skeletal actin promoter (HSA). Tamoxifen-induced Cre LoxP recombination was activated by oral administration of tamoxifen-containing chow (Tam400/Cre ER Harlan), which was administered ad libitum for 5 weeks. Muscles were collected 5 weeks after the tamoxifen diet finished. Cre-negative littermates, also receiving tamoxifen treatment, were used as controls. Adult mice (3- to 5-month-old) of the same sex and age were used for each individual experiment. For fasting experiments, control animals were fed ad libitum; food pellets were removed from the cages of the fasted animals for $24 \mathrm{~h}$ before killing and muscles collection. PCR genotyping was performed with the following primers:

DRP1 Fw: CAGCTGCACTGGCTTCATGACTC

DRP1 Rv: GTCAACTTGCCATAAACCAGAG

Cre Fw: CACCAGCCAGCTATCAACTCG

Cre Rv: TTACATTGGTCCAGCCACCAG

Gene-expression analyses. The total RNA was prepared from gastrocnemius muscles or liver using TRIzol (Invitrogen). Complementary DNA was generated from $0.4 \mu \mathrm{g}$ of RNA reverse-transcribed with SuperScript III Reverse Transcriptase (Invitrogen). Duplicates of cDNA samples were then amplified on the 7900HT Fast Real-Time PCR System (Applied Biosystems) using the Power SYBR Green RT-PCR kit (Applied Biosystems). All data were normalized to GAPDH expression and plotted in arbitrary units as mean \pm SEM. The oligonucleotide primers used are shown in Supplementary Table 4.

IGF1 quantification was performed using TaqMan ${ }^{\circledR}$ Universal PCR Master Mix and the specific TaqMan primers IGF1 class II (Mm 00439559_m1, Life Technologies). The data were normalized to GAPDH expression (Mm 99999915_g1, Life Technologies). miR1 was quantified using single specific RTqPCR using TaqMan MicroRNA Assays (Applied Biosystems by Thermo Fisher Scientific, Waltham, MA, USA). cDNA synthesis was obtained starting from $5 \mu \mathrm{l}$ of RNA with the TaqMan MicroRNA Reverse Transcription Kit, and the amplification was subsequently performed with TaqMan Fast Universal PCR Master Mix using $1 \mu \mathrm{l}$ of cDNA and specific TaqMan primers (miR1 Assay ID 002222). The results are expressed as mean \pm SEM.

Immunoblotting. Muscles were powdered by pestle and mortar and lysed in a buffer containing $50 \mathrm{mM}$ Tris pH 7.5, $150 \mathrm{mM} \mathrm{NaCl}, 5 \mathrm{mM} \mathrm{MgCl} 2,1 \mathrm{mM}$ DTT, $10 \%$ glycerol, $2 \%$ SDS, $1 \%$ Triton X-100, Roche Complete Protease Inhibitor Cocktail, $1 \mathrm{mM}$ PMSF, $1 \mathrm{mM} \mathrm{NaVO}$, $5 \mathrm{mM} \mathrm{NaF}$, and $3 \mathrm{mM}$ b-glycerophosphate The lysis buffer used for MEF and C2C12 cells contained $50 \mathrm{mM}$ Tris $\mathrm{pH} 7.5,150$ $\mathrm{mM} \mathrm{NaCl}, 5 \mathrm{mM} \mathrm{MgCl}, 1 \mathrm{mM}$ DTT, $10 \%$ glycerol, $1 \mathrm{mM}$ EDTA, $0.5 \%$ Triton X100 , and the protease inhibitors listed above. Samples were immunoblotted and visualized with Super-Signal West Pico Chemiluminescent substrate (Pierce). Blots were stripped using Restore Western Blotting Stripping Buffer (Pierce) according to the manufacturer's instructions and reprobed if necessary. List of antibodies is depicted in Supplementary Table 5.

Uncropped blots are shown in Supplementary Fig. 10.

Nuclear protein extraction. Tibialis anterior muscles were lysed in $700 \mu \mathrm{l}$ of lysis buffer (10 mM HEPES, $10 \mathrm{mM} \mathrm{KCl,} 5 \mathrm{mM} \mathrm{MgCl} 2,0.5 \mathrm{mM}$ DTT, $1 \mathrm{mM}$ PMSF, 1 $\mathrm{mM}$ sodium vanadate, $50 \mathrm{mM}$ sodium fluoride, and $25 \mathrm{mM} \beta$-glycerophosphate) and then centrifuged to separate nuclear fraction from cytosolic fraction. Proteins from nuclear fraction were obtained after incubation for $10 \mathrm{~min}$ at $70^{\circ} \mathrm{C}, 46 \mathrm{~g}$, 
and centrifuged for $10 \mathrm{~min}, 15.871 \mathrm{~g}$. Proteins from the cytosolic fraction were precipitated overnight after the addition of cold acetone and centrifuged for $5 \mathrm{~min}$ at $12,000 \mathrm{~g}$.

Imaging and transmission EM. Cryosections of both P12 newborns hindlimb cross-sections and adult TA were stained for H\&E and SDH. The total myofiber numbers and CSA in conditional model were calculated from entire hindlimb cross-section based on assembled mosaic image $(\times 20$ magnification). Adult mice CSA was performed on TA as described ${ }^{29}$. For EM, EDL muscles were dissected from killed animals, pinned on a Sylgard dish, fixed at room temperature with $3.5 \%$ glutaraldehyde in $0.1 \mathrm{M}$ sodium cacodylate buffer $(\mathrm{NaCaCO})(\mathrm{pH} 7.4)$, and stored in the fixative at $4{ }^{\circ} \mathrm{C}$. Fixed muscles were then postfixed in a mixture of $2 \% \mathrm{OsO}_{4}$ and $0.8 \% \mathrm{~K}_{3} \mathrm{Fe}(\mathrm{CN})_{6}$ for $1-2 \mathrm{~h}$, rinsed with $0.1 \mathrm{M} \mathrm{NaCaCO}$ with $75 \mathrm{mM} \mathrm{CaCl}_{2}$, enblock stained with saturated uranyl acetate, and embedded for EM in epoxy resin (Epon 812$)$ as in ref. ${ }^{48}$. Ultrathin sections $(\sim 40 \mathrm{~nm})$ were cut in a Leica Ultracut $\mathrm{R}$ microtome (Leica Microsystem, Austria) using a Diatome diamond knife (DiatomeLtd. CH-2501 Biel, Switzerland) and examined at $60 \mathrm{kV}$ after double-staining with uranyl acetate and lead citrate, with a FP 505 Morgagni Series 268D electron microscope (FEI Company, Brno, Czech Republic), equipped with Megaview III digital camera (Munster, Germany) and Soft Imaging System (Germany).

Quantitative analyses of the mitochondria. The data contained in Supplementary Table 1 and Supplementary Table 2 were collected from 5 to 6-month-old wild-type (WT, $n=3$ mice) and Drp1-KO $(n=4$ mice) EDL muscles. Micrographs of non-overlapping regions were randomly collected from transversal and longitudinal sections of internal fiber areas of EDL muscles. Sample size is detailed in the Supplementary Table legend.

Mitochondrial volume (Supplementary Table 1, column A and Supplementary Fig. 2a) was determined using the well-established stereology point-counting technique ${ }^{49,50}$ in micrographs taken from transversal sections at $\times 7.100$ magnifications. Briefly, after superimposing an orthogonal array of dots at a spacing of $0.20 \mu \mathrm{m}$ to the electron micrographs, the ratio between numbers of dots falling within mitochondrial profiles and the total number of dots covering the whole image was used to calculate the relative fiber volume occupied by the mitochondria.

The number of the mitochondria and CRUs/area (Supplementary Table 1, column B; Supplementary Table 2 column A) was evaluated in micrographs taken at $\times 14.000$ magnifications in longitudinal sections and reported as average number over $100 \mu \mathrm{m}^{2}$. If an individual mitochondrion extended from one I band to another, it was counted in both. In each EM image, we also determined (i) orientation (oblique/longitudinal triads) of CRUs; (ii) morphology (dyads) of CRUs expressed as percentages over the total number of CRUs; (iii) the number of mitochondria-CRUs pairs/area (Supplementary Table 2, columns B, C, D, respectively).

The number of severely damaged mitochondria (Supplementary Table 1 , column C) was evaluated in micrographs of longitudinal sections taken at $\times 14.000$ magnifications; their number is reported as average number over $100 \mu \mathrm{m}^{2}$ and as the percentage of the total number (in parenthesis). Mitochondria with any or several of the following ultrastructural alterations were classified as severely damaged: (a) presenting disruption of the external membrane; (b) presence of internal vacuolization and/or disrupted internal cristae; and (c) containing myelinfigures.

The average area of the mitochondria (Supplementary Table 1, column D and Supplementary Fig. 2a) was measured in the same set of micrographs using the Soft Imaging System (Germany) provided with the electron microscope. Only mitochondria which were entirely visualized in the micrograph were measured.

Measurement of mitochondrial DNA copy number. The total gastrocnemius DNA was isolated using Puregene Cell and Tissue Kit (Qiagen) and was amplified using specific primers for mtCOXII and $18 \mathrm{~S}$ by real-time PCR using the Power SYBR Green RT-PCR kit (Applied Biosystems). The mtDNA copy number was calculated using $18 \mathrm{~S}$ amplification as a reference for nuclear DNA content. Realtime PCR was performed with the following primers:

18S Fw: CATTCGAACGTCTGCCCTATCA

18S Rw: GGGTCGGGAGTGGGTAATTTG

COXII Fw: GCCGACTAAATCAAGCAACA

COXII Rw: CAATGGGCATAAAGCTATGG

Mitochondrial assays. Muscle mitochondria from the indicated genotype were isolated by differential centrifugation steps ${ }^{51}$. To detect RCS, Blue Native PAGE was performed by resupending mitochondrial pellets in Native Buffer (Invitrogen) plus $4 \%$ Digitonin (SIGMA) to a final concentration of $10 \mu \mathrm{g} / \mu \mathrm{l}$ and incubated for $1 \mathrm{~h}$ on ice. After $20 \mathrm{~min}$ of centrifugation at $16,000 \mathrm{~g}$, the supernatant was collected and one-third of digitonin percentage of sample buffer 5\% G250 (Invitrogen) was added. Then $50 \mu \mathrm{g}$ of mitochondrial membrane proteins were loaded and run on a $3-12 \%$ Bis-Tris gel (Invitrogen), as described in the NativePAGE ${ }^{\mathrm{m}}$ Novex $^{\circledast}$ Bis-Tris Gel System manual. Western blot was performed using a Trans-Blot transfer cell (Bio-Rad) at $4{ }^{\circ} \mathrm{C}$ overnight.
To measure respiration, mitochondria $(1 \mathrm{mg} / \mathrm{ml})$ were incubated in experimental buffer (EB: $150 \mathrm{mM} \mathrm{KCl,} 10 \mathrm{mM}$ Tris Mops, $10 \mathrm{mM}$ EGTA-Tris, and $10 \mathrm{mM}$ ATP). When indicated, mitochondria were transferred into a Clark's type oxygen electrode chamber and $5 \mathrm{mM}$ glutamate $/ 2.5 \mathrm{mM}$ malate or $2 \mathrm{mM}$ rotenone/ $10 \mathrm{mM}$ succinate or $3 \mathrm{mM}$ ascorbate/ $10 \mathrm{mM}$ TMPD were added. Basal $\mathrm{O}_{2}$ consumption was recorded (state 2), and after $2 \mathrm{~min} 100 \mathrm{mM}$ ADP was added (state 3), followed by $2.5 \mathrm{mg} / \mathrm{ml}$ oligomycin (state 4 ) and $200 \mathrm{mM}$ FCCP (state 3).

Assesment of mitochondrial respiratory chain enzymatic activities on muscles was determined spectrophotometrically ${ }^{52}$. The results were normalized to citrate synthase enzymatic activity.

Mitochondria oxidative stress measurements. Mitochondrial targeted HyPer (Hydrogen Peroxide sensor) was used as an indicator of mitochondrial redox status. Briefly, adult FDB muscles were transfected by electroporation with $\mathrm{mt}-\mathrm{HyPer}$ plasmid. After 7 days of transfection, single-muscle fibers were isolated from the control and knockout mice. Mt-HyPer fluorescence (excitation: 420 and $488 \mathrm{~nm}$, emission: $515 \mathrm{~nm}$ ) was measured for 8 min every $10 \mathrm{~s}$ and upon treatment with $\mathrm{H}_{2} \mathrm{O}_{2}$. The ratio of fluorescence intensities was determined by ImageJ Software.

Mitochondrial membrane potential determination. Mitochondrial membrane potential was measured in isolated fibers from flexor digitorum brevis (FDB) muscles, as previously described 29,53 . Briefly, FDB myofibers were placed in $1 \mathrm{ml}$ Tyrode's buffer and loaded with $2.5 \mathrm{nM}$ TMRM (Molecular Probes) supplemented with $1 \mu \mathrm{M}$ cyclosporine $\mathrm{H}$ (a P-glycoprotein inhibitor) for $30 \mathrm{~min}$ at $37^{\circ} \mathrm{C}$. Myofibers were then observed at a Olympus IMT-2 inverted microscope (Melville, NY) equipped with a CellR imaging system. Sequential images of TMRM fluorescence were acquired every $60 \mathrm{~s}$ with a $20 \times 0.5$ UPLANSL N A objective (Olympus). At the times indicated by arrows, oligomycin $(\mathrm{Olm}, 5 \mu \mathrm{M})$ (Sigma) or the protonophore carbonyl cyanide p-trifluoromethoxyphenylhydrazone (FCCP, $4 \mu \mathrm{M}$ ) (Sigma) was added to the cell culture medium. We used oligomycin to inhibit ATPsynthase, because ATP-synthase can reversely transport protons across the inner mitochondrial membrane, so maintaining the potential also in dysfunctional mitochondria. For this reason, only this treatment allows us to detect real dysfunctional mitochondria that would inevitably dissipate the potential, losing TMRM signal. Images were acquired, stored, and then analysis of TMRM fluorescence over mitochondrial regions of interest was performed using ImageJ software (http://rsb.info.nih.gov/ij/).

Free calcium concentration in the cytosol. FDB fibers were dissociated with collagenase were loaded with $5 \mu \mathrm{M}$ Fura-2 AM (Molecular Probes, Invitrogen) in incubation buffer $\left(125 \mathrm{mM} \mathrm{NaCl}, 5 \mathrm{mM} \mathrm{KCl}, 1 \mathrm{mM} \mathrm{MgSO}_{4}, 1 \mathrm{Mm} \mathrm{KH}_{2} \mathrm{PO}_{4}, 5.5\right.$ $\mathrm{mM}$ glucose, $1 \mathrm{mM} \mathrm{CaCl}$, $20 \mathrm{Mm}$ HEPES, and $1 \%$ bovine serum albumin, $\mathrm{pH}$ adjusted to 7.4 with $\mathrm{NaOH}$ ), washed twice for 10 min with incubation buffer without $\mathrm{BSA}$ at $37^{\circ} \mathrm{C}$ to retain the indicator in the cytosol, and immersed in imaging buffer $\left(125 \mathrm{mM} \mathrm{NaCl}, 5 \mathrm{mM} \mathrm{KCl}, 1 \mathrm{mM} \mathrm{CaCl} 2,1 \mathrm{mM} \mathrm{MgSO}_{4}, 1 \mathrm{mM}\right.$ $\mathrm{KH}_{2} \mathrm{PO}_{4}, 5 \mathrm{mM}$ glucose, $20 \mathrm{mM}$ HEPES). After a minimum of $30 \mathrm{~min}$, calcium signals were recorded using a dual-beam excitation fluorescence photometry setup (IonoOptix Corp.) at $25^{\circ} \mathrm{C}$. Fibers were electrically or chemically stimulated following the protocol described below. $\mathrm{Ca}^{2+}$ measurements were expressed as fluorescence ratio of the emission at $480 \mathrm{~nm}$ with reference to the excitation wavelengths of 360 and $380 \mathrm{~nm}$, respectively.

To study calcium release under physiological condition of contractile activity, fibers were treated with BTS $20 \mu \mathrm{M}$ to block spontaneous contraction, then electrically stimulated with trains of pulses at $0.5 \mathrm{~Hz}$, followed by stimulation trains at higher frequency $(60 \mathrm{~Hz}, 2 \mathrm{~s})$.

Chemical release of calcium from the SR was induced by sequential administration of caffeine and thapsigargin. Caffeine $20 \mathrm{mM}$ stimulates $\mathrm{Ca}^{2+}$ release from the SR through its action on RyR. Simultaneously, fibers were treated with thapsigargin $1 \mu \mathrm{M}$, an inhibitor of the SR/ER $\mathrm{Ca}^{2+}$ ATPase (SERCA).

Calibration of the Fura-2 AM signals and calculation of the free cytosolic calcium concentration: muscle fiber fluorescence $\mathrm{R}$ was converted to free cytosolic calcium concentration $\left[\mathrm{Ca}^{2+}\right] \mathrm{CY}$ using the equation $\left[\mathrm{Ca}^{2+}\right] \mathrm{CY}=\left(\mathrm{R}-\mathrm{R}_{\min }\right) /$ $\left(\mathrm{R}_{\max }-\mathrm{R}\right) \cdot \mathrm{KD} \cdot \beta$, where $\mathrm{R}$ is the ratio of fluorescence excited at $340 \mathrm{~nm}$ to that excited at $380 \mathrm{~nm}$ and $\mathrm{KD}$ is the affinity constant of Fura-2 for calcium, which was taken at $145 \mathrm{nM}$ (Molecular Probes). $\mathrm{R}_{\min }$ and $\mathrm{R}_{\max }$ were determined in ionopermeabilized myofibers: $R_{\min }$ was the minimum $R$ value measured in calcium-free solution, and $R_{\max }$ was the maximal $\mathrm{R}$ value measured in $2 \mathrm{mM}$ calcium solution. $\beta$ was derived as the $F 380$ calcium-free/F380 calcium-saturated R. Under these assumptions, the resting cytosolic calcium concentration will be $71 \mathrm{nM}$ at $\mathrm{R}=0.87$ and $87 \mathrm{nM}$ at $\mathrm{R}=0.92$.

Detection of free $\mathrm{Ca}^{2+}$ in the SR and mitochondrial matrix. Free $\mathrm{Ca}^{2+}$ con centrations in the mitochondrial matrix and SR lumen were determined using cameleon $\mathrm{Ca}^{2+}$ probes, $4 \mathrm{mtD} 3 \mathrm{cpv}^{54}$ for mitochondria and D1-ER ${ }^{55}$ for sarcoplasmic reticulum, kindly donated by Dr. R.Y. Tsien (University of California, San Diego)

The probes were expressed in FDB muscles by electroporation of plasmid vectors as described in the In vivo FDB electroporation section. 
Mitochondrial and the SR-free $\mathrm{Ca}^{2+}$ levels were determined from the YFP/CFP ratio of the emitted lights of the cameleon probes excited with UV light in an inverted fluorescence microscope (Eclipse-Ti; Nikon Instruments). Fibers were placed in chamber containing imaging buffer on the movable stage of the microscope, and temperature was set at $25^{\circ} \mathrm{C}$. CFP fluorophores were excited by means of a $\mathrm{Hg}$ arc lamp using a $435-\mathrm{nm}$ filter. CFP and YFP intensities were recorded by means of a cooled CCD camera (C9100-13; Hamamatsu), equipped with a dichroic mirror, at $535 \mathrm{~nm}$ (YFP emission) and $480 \mathrm{~nm}$ (CFP emission), respectively. Two images of $256 \times 128$ pixels each, corresponding, respectively, to YFP and CFP light emissions, were collected with a time resolution of $9 \mathrm{~ms}$. YFP and CFP intensities were corrected for background and the ratio $\mathrm{R}$ was defined as follows: $\mathrm{R}=\left(\mathrm{YFP}_{\text {fiber }}-\mathrm{YFP}_{\text {background }}\right) /\left(\mathrm{CFP}_{\text {fiber }}-\mathrm{CFP}_{\text {background }}\right)$.

Electrically induced release of calcium from the SR was achieved by electrical stimulation, as reported above (trains of $2 \mathrm{~s}$ at $60 \mathrm{~Hz}$ ). Chemical release of $\mathrm{Ca}^{2+}$ from the SR was induced by sequential administration of caffeine and thapsigargin. Caffeine $20 \mathrm{mM}$ stimulates $\mathrm{Ca} 2+$ release from SR through its action on RyR. Simultaneously, fibers were treated with thapsigargin $1 \mu \mathrm{M}$, an inhibitor of the sarco/endoplasmic reticulum $\mathrm{Ca} 2+$ ATPase (SERCA).

Cytosolic $\mathrm{Ca}^{2+}$ real-time imaging in MCU knocked down fibers. For real-time imaging, FDB muscles were digested in collagenase A ( $4 \mathrm{mg} / \mathrm{ml}$ ) (Roche) dissolved in Tyrode's salt solution ( $\mathrm{pH}$ 7.4) (Sigma-Aldrich) containing 10\% FBS (Thermo Fisher Scientific). Single fibers were isolated, plated on laminin-coated glass coverslips, and cultured in the DMEM with HEPES (Thermo Fisher Scientific), supplemented with $10 \% \mathrm{FBS}$. Fibers were maintained in culture at $37^{\circ} \mathrm{C}$ with $5 \% \mathrm{CO}_{2}$.

During the experiments, myofibers were maintained in Krebs-Ringer modified buffer ( $135 \mathrm{mM} \mathrm{NaCl}, 5 \mathrm{mM} \mathrm{KCl}, 1 \mathrm{mM} \mathrm{MgCl} 2,20 \mathrm{mM}$ HEPES, $1 \mathrm{mM} \mathrm{MgSO}_{4}, 0.4$ $\mathrm{mM} \mathrm{KH} \mathrm{KO}_{4}, 5.5 \mathrm{mM}$ glucose, $\mathrm{pH}$ 7.4) in the presence of $500 \mu \mathrm{M}$ EGTA and 75 $\mu \mathrm{M}$ BTS (Tocris). In total, $20 \mathrm{mM}$ caffeine and $1 \mu \mathrm{M}$ thapsigargin (Sigma-Aldrich) were added when indicated to elicit $\mathrm{Ca}^{2+}$ release from intracellular stores with no consequent reuptake. Experiments were performed on a Zeiss Axiovert 200 microscope equipped with a $40 \times / 1.3$ N.A. PlanFluor objective. Excitation was performed with a DeltaRAM V high-speed monochromator (Photon Technology International) equipped with a $75 \mathrm{~W}$ xenon arc lamp. Images were captured with a high-sensitivity Evolve 512 Delta EMCCD (Photometrics). The system is controlled by MetaMorph 7.5 (Molecular Devices) and was assembled by Crisel Instruments. FURA2-AM was excited every second at 340 and $380 \mathrm{~nm}$, respectively, and images were acquired through a 515/20 bandpass emission filter (Chroma). Exposure time was set to $50 \mathrm{~ms}$. Acquisition was performed at binning 1 with 200 of EM gain. Image analysis was performed with Fiji distribution of the ImageJ software ${ }^{56}$. Images were background subtracted, and $\Delta R / R_{0}$ was calculated. The data are expressed as area below curve integrating $150 \mathrm{~s}$ after stimulation.

Mito-mKeima mitophagy assay. Mitochondria-targeted mKeima plasmid (mtKeima) (MBL International) was used to monitor mitophagy in transfected FDB single fibers. mt-Keima is a coral-derived protein that exhibits both $\mathrm{pH}$-dependent excitation and resistance to lysosomal proteases. These properties allow rapid determinations to whether the protein is in mitochondria or the in lysosome $e^{57,58}$. In fluorescence microscopy, ionized Keima is detected as a red fluorescent signal at acidic $\mathrm{pH}$ (lysosome), and neutral Keima as a green fluorescent signal at higher $\mathrm{pH}$ (mitochondria) ${ }^{59}$. Fluorescence of mt-Keima was imaged in two channels via two sequential excitations $(458 \mathrm{~nm}$, green; $561 \mathrm{~nm}$, red) and using a $570-$ to $695-$ $\mathrm{nm}$ emission range. The level of mitophagy was defined as the total number of red pixels divided by the total number of all pixels.

In vivo protein synthesis measurements. In vivo protein synthesis was measured by using the SUnSET technique ${ }^{25,60}$. Mice were anesthetized and then given an intraperitoneal injection of $0.040 \mu \mathrm{mol} / \mathrm{g}$ puromycin dissolved in $100 \mu \mathrm{l}$ of PBS. At exactly $30 \mathrm{~min}$ after injection, muscles were collected and frozen in liquid $\mathrm{N}_{2}$ for western blot analysis. A mouse IgG2a monoclonal anti-puromycin antibody (clone 12D10, 1:5000) was used to detect puromycin incorporation.

Contractile force measurements. In vivo gastrocnemius force measurements were performed as described previously ${ }^{61}$. Briefly, mice were anesthetized, and stainless-steel electrodes wires were placed on either side of the sciatic nerve. Torque production of the plantar flexors was measured using a muscle lever system (Model 305c; Aurora Scientific, Aurora, ON, Canada). The force-frequency curves were determined by increasing the stimulation frequency in a stepwise manner, pausing for $30 \mathrm{~s}$ between stimuli to avoid effects due to fatigue. After force measurements, animals were killed by cervical dislocation and muscles were dissected and weighted. Force was normalized to the muscle mass as an estimate of specific force.

Ex vivo, EDL and SOL muscles were dissected in warm oxygenated Krebs solution and mounted between a force transducer (SI-H Force Transducer World Precision Instruments, Inc., Sarasota, FL, USA) and a micromanipulator-controlled shaft in a small chamber where oxygenated Krebs solution was continuously circulated. The temperature was kept constant at $25^{\circ} \mathrm{C}$.

After optimizing the stimulation conditions and muscle length, the responses to a single stimulus (twitch) or to a series of stimuli at increasing rates producing unfused or fused tetani were recorded. Time-to-peak tension, time-to-half relaxation, and peak-tension were measured in single twitches. Tension was measured in unfused and fused maximal tetani. Fatigue was induced by $120 \mathrm{~s}$ of repetitive stimulation applying every $2 \mathrm{~s}$ a train of $0.5 \mathrm{~s}$ duration (duty ratio $1 / 4$ ).

In vivo FDB electroporation. Electroporation experiments were performed on FDB muscles from wild-type and knockout animals. The animals were anesthetized by an intraperitoneal injection of xylazine (Xilor) $(20 \mathrm{mg} / \mathrm{Kg})$ and Zoletil $(10 \mathrm{mg} /$ $\mathrm{Kg}) .7 \mu \mathrm{l}$ of hyaluronidase $(2 \mathrm{mg} / \mathrm{ml}$ ) (Sigma-Aldrich) were injected in the feet of anesthetized mice to soften muscle tissue underneath the epidermis. After $50 \mathrm{~min}$ we injected $15 \mu \mathrm{g}$ of plasmid DNA (4mitoD3cpv Cameleon; mt-Keima probe; D1ER Cameleon; shRNA against MCU; DRP1-YFP) and after 10 min electric pulses were applied by two stainless needles placed at $1 \mathrm{~cm}$ from each other $(100 \mathrm{~V} / \mathrm{cm})$ $(100$ Volts $/ \mathrm{cm}, 20$ pulses, $1 \mathrm{~s}$ intervals). Muscles were analyzed 10 days later. No evidence of necrosis or inflammation were observed after the transfection procedure.

Autophagic flux quantification. We monitored autophagic flux in basal condition using colchicine ${ }^{62}$. Briefly, inducible transgenic mice were treated with $0.4 \mathrm{mg} / \mathrm{kg}$ of colchicine or the vehicle by intraperitoneal injection. The treatment was repeated twice every $12 \mathrm{~h}$ prior to muscle harvesting.

Plasma FGF21 and IGF1 measurements. Plasma was obtained from blood collected from controls and DRP1-null mice. Blood FGF21 levels were determined using rat/mouse FGF21 enzime-linked immunosorbent ELISA-Kit (Merck Millipore, EZRMFGF21-26K). Blood IGF1 levels were measured using mouse/rat IGF1 immunoassay (MG100, R\&D Systems) and following the manufacturer's instructions. The data are expressed in $\mathrm{pg} / \mathrm{ml}$.

Statistical analysis. All data are expressed as means \pm SEM of independent experiments. Statistical analysis was performed using two-tailed Student's $t$ test or two-way analysis of variant (ANOVA). When ANOVA revealed significant differences, further analysis was performed using Bonferroni's multiple comparison test. Differences between groups were considered statistically significant for $p<0.05$.

Reporting summary. Further information on research design is available in the Nature Research Reporting Summary linked to this article.

\section{Data availability}

The authors declare that all data supporting the findings of this study are available within the paper and/ or its supplementary information files.

Received: 27 November 2017 Accepted: 23 April 2019

Published online: 12 June 2019

\section{References}

1. Romanello, V. \& Sandri, M. Mitochondrial quality control and muscle mass maintenance. Front Physiol. 6, 422 (2015).

2. Kasahara, A. \& Scorrano, L. Mitochondria: from cell death executioners to regulators of cell differentiation. Trends Cell Biol. 24, 761-770 (2014).

3. Vainshtein, A., Grumati, P., Sandri, M. \& Bonaldo, P. Skeletal muscle, autophagy, and physical activity: the menage a trois of metabolic regulation in health and disease. J. Mol. Med. (Berl.) 92, 127-137 (2014).

4. LoVerso, F., Carnio, S., Vainshtein, A. \& Sandri, M. Autophagy is not required to sustain exercise and PRKAA1/AMPK activity but is important to prevent mitochondrial damage during physical activity. Autophagy 10, 1883-1894 (2014).

5. Otera, H. \& Mihara, K. Molecular mechanisms and physiologic functions of mitochondrial dynamics. J. Biochem. 149, 241-251 (2011).

6. Romanello, V. \& Sandri, M. Mitochondrial biogenesis and fragmentation as regulators of protein degradation in striated muscles. J. Mol. Cell Cardiol. $\mathbf{5 5}$, 64-72 (2013).

7. Smirnova, E., Griparic, L., Shurland, D. L. \& van der Bliek, A. M. Dynaminrelated protein Drp1 is required for mitochondrial division in mammalian cells. Mol. Biol. Cell 12, 2245-2256 (2001).

8. Ishihara, N. et al. Mitochondrial fission factor Drp1 is essential for embryonic development and synapse formation in mice. Nat. Cell Biol. 11, 958-966 (2009)

9. Wakabayashi, J. et al. The dynamin-related GTPase Drp1 is required for embryonic and brain development in mice. J. Cell Biol. 186, 805-816 (2009).

10. Romanello, V. et al. Mitochondrial fission and remodelling contributes to muscle atrophy. EMBO J. 29, 1774-1785 (2010).

11. Touvier, T. et al. Muscle-specific Drp1 overexpression impairs skeletal muscle growth via translational attenuation. Cell Death Dis. 6, e1663 (2015). 
12. Peterson, C. M., Johannsen, D. L. \& Ravussin, E. Skeletal muscle mitochondria and aging: a review. J. Aging Res. 2012, 194821 (2012).

13. Tezze, C. et al. Age-associated loss of OPA1 in muscle impacts muscle mass, metabolic homeostasis, systemic inflammation, and epithelial senescence. Cell Metab. 25, 1374-1389 e1376 (2017).

14. Zhou, J. et al. Changes in macroautophagy, chaperone-mediated autophagy, and mitochondrial metabolism in murine skeletal and cardiac muscle during aging. Aging (Albany NY) 9, 583-599 (2017).

15. Ibebunjo, C. et al. Genomic and proteomic profiling reveals reduced mitochondrial function and disruption of the neuromuscular junction driving rat sarcopenia. Mol. Cell Biol. 33, 194-212 (2013).

16. Barreto, R. et al. Cancer and chemotherapy contribute to muscle loss by activating common signaling pathways. Front Physiol. 7, 472 (2016).

17. van der Ende, M. et al. Mitochondrial dynamics in cancer-induced cachexia. Biochimica et biophysica acta. Rev. cancer 1870, 137-150 (2018).

18. Brown, J. L. et al. Mitochondrial degeneration precedes the development of muscle atrophy in progression of cancer cachexia in tumour-bearing mice. J. Cachex-. Sarcopenia Muscle 8, 926-938 (2017).

19. Song, J. et al. Effect of Qiangji Jianli decoction on mitochondrial respiratory chain activity and expression of mitochondrial fusion and fission proteins in myasthenia gravis rats. Sci. Rep. 8, 8623 (2018).

20. Bartsakoulia, M. et al. A novel mechanism causing imbalance of mitochondrial fusion and fission in human myopathies. Hum. Mol. Genet 27, 1186-1195 (2018).

21. Bothe, G. W., Haspel, J. A., Smith, C. L., Wiener, H. H. \& Burden, S. J. Selective expression of Cre recombinase in skeletal muscle fibers. Genesis 26, 165-166 (2000).

22. Cogliati, S. et al. Mitochondrial cristae shape determines respiratory chain supercomplexes assembly and respiratory efficiency. Cell 155, 160-171 (2013).

23. Mopert, K. et al. Loss of Drp1 function alters OPA1 processing and changes mitochondrial membrane organization. Exp. Cell Res. 315, 2165-2180 (2009).

24. Schiaffino, S., Dyar, K. A., Ciciliot, S., Blaauw, B. \& Sandri, M. Mechanisms regulating skeletal muscle growth and atrophy. FEBS J. 280, 4294-4314 (2013).

25. Schmidt, E. K., Clavarino, G., Ceppi, M. \& Pierre, P. SUnSET, a nonradioactive method to monitor protein synthesis. Nat. Methods 6, 275-277 (2009).

26. Milan, G. et al. Regulation of autophagy and the ubiquitin-proteasome system by the FoxO transcriptional network during muscle atrophy. Nat. Commun. 6 , 6670 (2015).

27. Sandri, M. et al. Foxo transcription factors induce the atrophy-related ubiquitin ligase atrogin-1 and cause skeletal muscle atrophy. Cell 117, 399-412 (2004).

28. Sartori, R. et al. BMP signaling controls muscle mass. Nat. Genet $\mathbf{4 5}$, 1309-1318 (2013).

29. Mammucari, C. et al. FoxO3 controls autophagy in skeletal muscle in vivo. Cell Metab. 6, 458-471 (2007)

30. Boncompagni, S. et al. Mitochondria are linked to calcium stores in striated muscle by developmentally regulated tethering structures. Mol. Biol. Cell 20, 1058-1067 (2009).

31. Grumati, P. et al. Autophagy is defective in collagen VI muscular dystrophies, and its reactivation rescues myofiber degeneration. Nat. Med. 16, 1313-1320 (2010).

32. Bertaggia, E., Coletto, L. \& Sandri, M. Posttranslational modifications control FoxO3 activity during denervation. Am. J. Physiol. Cell Physiol. 302, C587-C596 (2012).

33. Winbanks, C. E. et al. The bone morphogenetic protein axis is a positive regulator of skeletal muscle mass. J. Cell Biol. 203, 345-357 (2013).

34. Masiero, E. et al. Autophagy is required to maintain muscle mass. Cell Metab. 10, 507-515 (2009).

35. Zaglia, T. et al. Content of mitochondrial calcium uniporter (MCU) in cardiomyocytes is regulated by microRNA-1 in physiologic and pathologic hypertrophy. Proc. Natl Acad. Sci. USA 114, E9006-E9015 (2017).

36. Gutierrez, T. \& Simmen, T. Endoplasmic reticulum chaperones tweak the mitochondrial calcium rheostat to control metabolism and cell death. Cell Calcium 70, 64-75 (2018).

37. Debattisti, V., Pendin, D., Ziviani, E., Daga, A. \& Scorrano, L. Reduction of endoplasmic reticulum stress attenuates the defects caused by Drosophila mitofusin depletion. J. Cell Biol. 204, 303-312 (2014).

38. Mammucari, C. et al. The mitochondrial calcium uniporter controls skeletal muscle trophism in vivo. Cell Rep. 10, 1269-1279 (2015).

39. Gimeno, R. E. \& Moller, D. E. FGF21-based pharmacotherapy-potential utility for metabolic disorders. Trends Endocrinol. Metab. 25, 303-311 (2014).

40. Kim, K. H. et al. Autophagy deficiency leads to protection from obesity and insulin resistance by inducing Fgf21 as a mitokine. Nat. Med. 19, 83-92 (2013).

41. Boncompagni, S. et al. Characterization and temporal development of cores in a mouse model of malignant hyperthermia. Proc. Natl Acad. Sci. USA 106, 21996-22001 (2009).
42. Rudolf, R., Mongillo, M., Magalhaes, P. J. \& Pozzan, T. In vivo monitoring of $\mathrm{Ca}(2+)$ uptake into mitochondria of mouse skeletal muscle during contraction. J. Cell Biol. 166, 527-536 (2004).

43. De Stefani, D., Raffaello, A., Teardo, E., Szabo, I. \& Rizzuto, R. A fortykilodalton protein of the inner membrane is the mitochondrial calcium uniporter. Nature 476, 336-340 (2011).

44. Giorgio, V., Guo, L., Bassot, C., Petronilli, V. \& Bernardi, P. Calcium and regulation of the mitochondrial permeability transition. Cell Calcium $\mathbf{7 0}$, 56-63 (2017)

45. Millay, D. P. et al. Genetic and pharmacologic inhibition of mitochondrialdependent necrosis attenuates muscular dystrophy. Nat. Med. 14, 442-447 (2008).

46. Irwin, W. A. et al. Mitochondrial dysfunction and apoptosis in myopathic mice with collagen VI deficiency. Nat. Genet. 35, 367-371 (2003).

47. Angelin, A. et al. Mitochondrial dysfunction in the pathogenesis of Ullrich congenital muscular dystrophy and prospective therapy with cyclosporins. Proc. Natl Acad. Sci. USA 104, 991-996 (2007).

48. Pietrangelo, L. et al. Age-dependent uncoupling of mitochondria from Ca2 $(+)$ release units in skeletal muscle. Oncotarget 6, 35358-35371 (2015).

49. Loud, A. V., Barany, W. C. \& Pack, B. A. Quantitative evaluation of cytoplasmic structures in electron micrographs. Lab. Invest. 14, 996-1008 (1965).

50. Mobley, B. A. \& Eisenberg, B. R. Sizes of components in frog skeletal muscle measured by methods of stereology. J. Gen. Physiol. 66, 31-45 (1975).

51. Frezza, C., Cipolat, S. \& Scorrano, L. Organelle isolation: functional mitochondria from mouse liver, muscle and cultured fibroblasts. Nat. Protoc. 2, 287-295 (2007)

52. Spinazzi, M., Casarin, A., Pertegato, V., Salviati, L. \& Angelini, C. Assessment of mitochondrial respiratory chain enzymatic activities on tissues and cultured cells. Nat. Protoc. 7, 1235-1246 (2012).

53. Zhao, J. et al. FoxO3 coordinately activates protein degradation by the autophagic/lysosomal and proteasomal pathways in atrophying muscle cells. Cell Metab. 6, 472-483 (2007).

54. Giacomello, M. et al. $\mathrm{Ca}^{2+}$ hot spots on the mitochondrial surface are generated by $\mathrm{Ca} 2+$ mobilization from stores, but not by activation of storeoperated Ca2+ channels. Mol. Cell 38, 280-290 (2010).

55. Palmer, A. E., Jin, C., Reed, J. C. \& Tsien, R. Y. Bcl-2-mediated alterations in endoplasmic reticulum $\mathrm{Ca} 2+$ analyzed with an improved genetically encoded fluorescent sensor. Proc. Natl Acad. Sci. USA 101, 17404-17409 (2004).

56. Schindelin, J. et al. Fiji: an open-source platform for biological-image analysis. Nat. Methods 9, 676-682 (2012).

57. Katayama, H., Kogure, T., Mizushima, N., Yoshimori, T. \& Miyawaki, A. A sensitive and quantitative technique for detecting autophagic events based on lysosomal delivery. Chem. Biol. 18, 1042-1052 (2011).

58. Sun, N. et al. Measuring in vivo mitophagy. Mol. Cell 60, 685-696 (2015).

59. Proikas-Cezanne, T. \& Codogno, P. A new fluorescence-based assay for autophagy. Chem. Biol. 18, 940-941 (2011).

60. Goodman, C. A. et al. Novel insights into the regulation of skeletal muscle protein synthesis as revealed by a new nonradioactive in vivo technique. FASEB J. 25, 1028-1039 (2011).

61. Blaauw, B. et al. Inducible activation of Akt increases skeletal muscle mass and force without satellite cell activation. Faseb J. 23, 3896-3905 (2009).

62. Ju, J. S., Varadhachary, A. S., Miller, S. E. \& Weihl, C. C. Quantitation of "autophagic flux" in mature skeletal muscle. Autophagy 6, 929-935 (2010).

\section{Acknowledgements}

We thank Laura Pietrangelo, $\mathrm{PhD}$ for technical help in preparing samples for EM. We are grateful to Dr. Asushi Miyawaki for the kind gift of mt-mKeima. This work was supported from the European Union ERC (282310-MyoPHAGY), AFM-Telethon (19524), Italian Ministry of Education (MiUR) (PRIN 2010/2011), Foundation Leducq, AIRC (17388), H2020-MSCA-RISE-2014 project no 645648 "Muscle Stress Relief”, and CARIPARO to M.S.; starting grants CARIPARO to V.R. and M.S.; Italian Telethon ONLUS Foundation (GGP13213) to F.P.; Italian Ministry of Health (GR-2011-02352681) to S.B.; Italian Telethon ONLUS Foundation GGP14187c, Fondazione CARIPARO and Fondazione IRP Città della Speranza to Leonardo Salviati; Italian Ministry of Education (MiUR) (PRIN 2015) to C.R., CARIPARO eccellenza and SNF 31003A_133130 to Luca Scorrano.

\section{Author contributions}

G.F. and V.R. generated muscle-specific DRP1 knockout mice. performed muscle transfections, mouse treatments, biochemical, morphological, immunohistochemical, and RNA analyses,. G.F., V.R., T.V., M.A.D., V.M., and M.A.,C.T. performed mitochondrial functional assays. M.C., G.G., D.dS., and C.M. performed in vivo calcium measurements. B. B. analyzed muscle mechanics. L.S. provided DRP1 floxed mice. S.B and F.P. performed electron microscopy. G.F., V.R., C.R., L. Scorrano, and L. Salviati supervised research and contributed to writing. G.F, V.R., and M.S. designed the study, analyzed the data, and wrote the paper. All authors discussed the results and corrected the paper. 


\section{Additional information}

Supplementary Information accompanies this paper at https://doi.org/10.1038/s41467019-10226-9.

Competing interests: The authors declare no competing interests.

Reprints and permission information is available online at http://npg.nature.com/ reprintsandpermissions/

Journal peer review information: Nature Communications thanks the anonymous reviewers for their contribution to the peer review of this work.

Publisher's note: Springer Nature remains neutral with regard to jurisdictional claims in published maps and institutional affiliations. (c) (i) Open Access This article is licensed under a Creative Commons Attribution 4.0 International License, which permits use, sharing, adaptation, distribution and reproduction in any medium or format, as long as you give appropriate credit to the original author(s) and the source, provide a link to the Creative Commons license, and indicate if changes were made. The images or other third party material in this article are included in the article's Creative Commons license, unless indicated otherwise in a credit line to the material. If material is not included in the article's Creative Commons license and your intended use is not permitted by statutory regulation or exceeds the permitted use, you will need to obtain permission directly from the copyright holder. To view a copy of this license, visit http://creativecommons.org/ licenses/by/4.0/.

(C) The Author(s) 2019 